\title{
Pricing and Semimartingale Representations of Vulnerable Contingent Claims in Regime-Switching Markets
}

\author{
Agostino Capponi* José E. Figueroa-López ${ }^{\dagger} \quad$ Jeffrey Nisen ${ }^{\ddagger}$
}

\begin{abstract}
Using a suitable change of probability measure, we obtain a novel Poisson series representation for the arbitragefree price process of vulnerable contingent claims in a regime-switching market driven by an underlying continuoustime Markov process. As a result of this representation, along with a short-time asymptotic expansion of the claim's price process, we develop an efficient method for pricing claims whose payoffs may depend on the full path of the underlying Markov chain. The proposed approach is applied to price not only simple European claims such as defaultable bonds, but also a new type of path-dependent claims that we term self-decomposable, as well as the important class of vulnerable call and put options on a stock. We provide a detailed error analysis and illustrate the accuracy and computational complexity of our method on several market traded instruments, such as defaultable bond prices, barrier options, and vulnerable call options. Using again our Poisson series representation, we show differentiability in time of the pre-default price function of European vulnerable claims, which enables us to rigorously deduce Feynman-Kač representations for the pre-default pricing function and new semimartingale representations for the price process of the vulnerable claim under both risk-neutral and objective probability measures.
\end{abstract}

AMS 2000 subject classifications: 93E20, 60J20.

Keywords and phrases: Credit Risk, Regime-Switching Models, Option Pricing, Vulnerable Claims, Semimartingale Representations.

\section{Introduction}

Regime-switching models are aimed at capturing the appealing idea that the macro-economy is subjected to regular, yet unpredictable in time, regimes, which in turn affect the prices of financial securities. For example, structural changes of macro-economic conditions, such as inflation and recession, may induce changes in the stock returns or in the term structure of interest rates and, similarly, periods of high market turbulence and liquidity crunches may increase the default risk of financial institutions. This has been empirically verified in the stock market, as stated by Ang and Bekaert (2002-b), who proposed the existence of two regimes characterized by different levels of volatility. Similar findings have also been documented in the bond market (see Ang and Bekaert (2002-a), Ang and Bekaert (2002-c), and Dai et al. (2007)). Most recently, in the credit market, Giesecke et al. (2011) suggested the existence of three regimes, associated with high, middle, and low default risk, via an empirical analysis of the corporate bond market over the course of the last 150 years. These considerations have led many researchers to use regime-switching models for asset pricing. In the context of options on stocks, Guo (2001) considered a market consisting of two regimes, and provided a semi-analytical formula for the option price, based on occupation time densities. Buffington and Elliott (2002) generalized the method to the case of multiple regimes, under the assumption that the generator of the Markov chain is time homogenous, and derived expressions for the price of European claims. Gapeev and Jeanblanc (2010)

\footnotetext{
*School of Industrial Engineering, Purdue University, West Lafayette, IN, 47907, USA (capponi@purdue.edu).

$\dagger$ Department of Statistics, Purdue University, West Lafayette, IN, 47907, USA (figueroa@purdue.edu).

$\ddagger$ Department of Statistics, Purdue University, West Lafayette, IN, 47907, USA (jnisen@purdue.edu).
} 
obtained closed form expressions for European claims, assuming geometric Brownian motion dynamics with regimeswitching drifts, while Yao et al. (2006) developed a fixed point iteration scheme to recover prices of European options in a regime-switching model, assuming time homogenous generators. Regime-switching models for default-free interest rates derivatives have been studied by Elliott and Wilson (2007), Elliott and Siu (2008), and Siu (2010-a). Norberg (2000) studied no-arbitrage pricing of derivatives on the regime parameters with emphasis on computation.

Most of the literature has focused on default-free European style claims with "simple" payoffs (i.e. payoffs depending only on the value of a random variable such as the terminal value of the underlying asset or the underlying source of randomness) with the exception of a few works detailed next. Guo and Zhang (2004) studied American-type default-free claims under regime-switching models. Graziano and Rogers (2006) provided a methodology to price barrier options with a regime-switching dividend process. In the context of credit risk, Siu (2010-b) considered pricing of interest rate derivatives with a defaultable counterparty. Siu et al. (2008) analyzed credit default swaps, and derived a system of coupled partial differential equations yielding the term structure of default probabilities needed for pricing. Bielecki et al. (2008-a) and Bielecki et al. (2008-b) studied pricing and hedging of a defaultable game option under a Markov modulated default intensity framework. Blanchet-Scalliet and Jeanblanc (2004) gave the hedging strategy of vulnerable claims using defaultable zero-coupon bonds and default-free assets, under the assumption that the default-free market is complete. Bielecki and Jeanblanc (2005) applied the theory of indifference pricing for pricing and replication of vulnerable claims within the reduced form framework. For an excellent survey on pricing and hedging aspects of vulnerable claims, the reader is referred to Bielecki et al. (2004).

In this paper, we consider a macro-economy with finitely many observable economic regimes, containing state information regarding the equity (drift and volatility), credit (hazard intensity and loss given default), and interest rate market (short rate). We consider three liquidly traded securities, namely, a money market account, a risky (default-free) stock, and a defaultable bond. The dynamics of the securities are assumed to depend on the macroeconomic regimes, which are modeled using a finite state continuous time Markov process. We follow the reduced form approach to credit risk and model the default event using a doubly stochastic framework. Pricing of contingent claims is still problematic in the context of regime-switching models, in part, due to the lack of easily computable expressions for option prices. Hence, explicit characterizations of these quantities that allow their efficient evaluation are of great need in the field. Our paper contributes to this fundamental problem by developing a novel approach for pricing vulnerable claims in a regime-switching model consisting of an arbitrary finite number of regimes, whose dynamics is governed by a possibly time varying generator. The proposed method can also be applied to price a certain type of path dependent claims, referred hereafter as self-decomposable claims, whose payoffs can be decomposed in terms of payoffs of shorter maturity claims, of possibly different type (see Section 5.1 for the precise statement). Self-decomposable claims encompass not only basic instruments, such as bonds, whose price may also be recovered from no-arbitrage arguments via the solution of a coupled system of ordinary differential equations (ODE's), but also more exotic pathdependent instruments, where prices can only be recovered via Monte-Carlo methods. As an example, we apply our algorithm to price a type of barrier options on the volatility of the stock, which turn out to be self-decomposable in terms of shorter maturity barrier options and bond prices. Note that, in our macro-economic model, our regime driven parameters for the short rate, volatility, and the default intensity may be seen as "quantized" proxies for the actual interest rates, volatilities, and credit spread indices, and, therefore, options on these underlying financial measurements provide means for the investor to hedge against interest rate risk, market and default risk, in different economic regimes.

The key tool behind our results is a type of Poisson series representation for the prices of vulnerable claims obtained by applying a novel change of probability measure technique, which transforms the underlying Markov process into a "homogenous" process with constant transition intensities. This Poisson representation enables us to express the price of the claim as a series expansion in terms of Laplace transforms of the symmetric Dirichlet distribution, which can be efficiently evaluated by Taylor approximations whenever the claim's maturity is small. Hence, a second important step in our method is to decompose the claim's price in terms of shorter dated claims. We provide a unified framework to quantify (and hence to control) the error due to the Taylor approximations, the error due to the discrete approximation of the time-varying generators, and even the error due to the truncation of the Poisson series representation. Our algorithm is computationally fast and, even for simple claims such as bonds, is able to achieve a high level of accuracy 
in the price, within a time complexity which compares favorably with standard ODE methods. As a far reaching application of our method, we also propose a new method to price vulnerable call/put options on the stock (where there is an additional risk factor represented by the Brownian motion), a subject which has received significant attention in the literature, as documented above.

Under mild assumptions on the Markov chain generator, we also apply our Poisson series representation to give a rigorous proof of the differentiability of the pre-default price function. This, in turn, allows us to provide Feynman-Kač and semimartingale representations for the pre-default price process (given by Eq. (9) below), and for the vulnerable claim price process (given by Eq. 10 below). This extends earlier results in the literature which, on one hand, had focused mostly on martingale representations and, on the other, typically presumed the time differentiability of the price function (e.g., Elliott and Kopp (2004), Elliott et al. (2007)). Needless to say that our semimartingale representations play a key role in the hedging of defaultable contingent claims, a problem which has attracted increasing attention since the start of the credit crisis, due to the high number of credit quality deteriorations, or even default events, experienced by financial institutions. Although we do not explicitly address the hedging problem in this paper, our formulas can be used within any hedging framework employing Markov-modulated dynamics. We refer the reader to Bielecki et al. (2003-a), Bielecki et al. (2003-b), Bielecki et al. (2004), Blanchet-Scalliet and Jeanblanc (2004), and Bielecki and Crepey (2011) for hedging methodologies based on the mean-variance or BSDE approach. The semimartingale representations are also fundamental in portfolio optimization problems as demonstrated in Capponi and Figueroa-López (2012). Another related work is Liew and Siu (2011), where a martingale representation for a two-dimensional martingale generated by the Brownian motion and the Markov chain was provided with the objective of establishing the existence of an admissible investment strategy in a default-free regime-switching market.

The rest of the paper is organized as follows. Section 2 sets up the defaultable regime-switching model. Section 3 establishes the Poisson series representation of the vulnerable claim price function. Section 4 provides rigorous semimartingale representations for the price process of the vulnerable claims. Section 5 presents our novel efficient method for pricing vulnerable claims and provides a rigorous error analysis. Section 6 illustrates the accuracy and computational power of our algorithms on concrete choices of claims. Section 7 concludes the paper. Proofs and numerical details are deferred to the Appendix.

\section{The defaultable regime-switching model}

As in Capponi and Figueroa-López (2012), we consider a regime-switching market model with a defaultable security driven by a continuous-time Markov process $X$. More specifically, our market consists of a risk-free asset, a risky (default-free) asset, and a defaultable $T$-claim (such as a bond) with respective price processes $\left\{B_{t}\right\}_{t \geq 0},\left\{S_{t}\right\}_{t \geq 0}$, and $\{\gamma(t, T)\}_{0 \leq t \leq T}$ defined on a complete filtered probability space $(\Omega, \mathcal{G}, \mathbb{G}, \mathbb{P})$. Here, $\mathbb{P}$ denotes the real world or historical probability measure and $\mathbb{G}:=\left(\mathcal{G}_{t}\right)$ is an enlarged filtration given by $\mathcal{G}_{t}:=\mathcal{F}_{t} \vee \mathcal{H}_{t}$, where $\mathbb{F}:=\left\{\mathcal{F}_{t}\right\}_{t \geq 0}$ models the reference filtration and $\mathcal{H}_{t}=\sigma(H(u): u \leq t)$ is the filtration generated by an exogenous default process $H(t):=\mathbf{1}_{\tau \leq t}$, after completion and regularization on the right (see Belanger et al. (2004) for details). Throughout, $\tau$ represents the default time of the defaultable security of interest. We also adopt the canonical construction of the default time $\tau$ in terms of a given hazard process $\left\{h_{t}\right\}_{t \geq 0}$, so that

$$
\tau:=\inf \left\{t \in \mathbb{R}_{+}: \int_{0}^{t} h_{u} d u \geq \Theta\right\}
$$

where $\Theta$ is an exponential random variable defined on the probability space $(\Omega, \mathcal{G}, \mathbb{P})$ and independent of $\mathbb{F}$. In that case, it follows that

$$
\xi_{t}^{\mathbb{P}}:=H(t)-\int_{0}^{t}\left(1-H\left(u^{-}\right)\right) h_{u} d u
$$

is a $\mathbb{G}$-martingale under $\mathbb{P}$ (see Bielecki and Rutkowski (2001), Section 6.5). The intuition of (2) is that one needs to compensate the single jump process for default prior to the occurrence of a default. Let us also recall that, under the 
previous setting, $\mathbb{F}$ satisfies the so-called martingale invariance property with respect to $\mathbb{G}$, which states that every $\mathbb{F}$ martingale is also a $\mathbb{G}$ martingale (see Sections 8.3.1 and 8.6.1 in Bielecki and Rutkowski (2001)). The previous principle is also typically referred to as the H-hypothesis.

As previously mentioned, we place ourselves in a regime-switching market model. More specifically, we define an $\mathbb{F}$-adapted continuous-time Markov process $\left\{X_{t}\right\}_{t \geq 0}$ with finite state space $\left\{e_{1}, e_{2}, \ldots, e_{N}\right\}$, where hereafter $e_{i}=$ $(0, \ldots, 1, \ldots 0)^{\prime} \in \mathbb{R}^{N}$ and ' denotes the transpose. Throughout, $p_{i, j}(t, s):=\mathbb{P}\left(X_{s}=e_{j} \mid X_{t}=e_{i}\right)$ for $t \leq s$ represents the transition probabilities of $X$ and $A(t):=\left[a_{i, j}(t)\right]_{i, j=1,2, \ldots, N}$ denotes the generator, defined by

$$
a_{i, j}(t)=\lim _{h \rightarrow 0} \frac{p_{i, j}(t, t+h)}{h}, \quad(i \neq j), \quad a_{i, i}(t):=-\sum_{j \neq i} a_{i, j}(t) .
$$

The following semimartingale representation is well-known (cf. Elliott et al. (1994)):

$$
X_{t}=X_{0}+\int_{0}^{t} A^{\prime}(s) X_{s} d s+M^{\mathbb{P}}(t)
$$

where $M^{\mathbb{P}}(t)=\left(M_{1}^{\mathbb{P}}(t), \ldots, M_{N}^{\mathbb{P}}(t)\right)^{\prime}$ is a $\mathbb{R}^{N}$-valued $\mathbb{P}$-martingale process. The following terminology will also be frequently needed in the sequel:

$$
C_{t}:=\sum_{i=1}^{N} i \mathbf{1}_{\left\{X_{t}=e_{i}\right\}} .
$$

We now specify the three instruments in the market, whose dynamics are driven by $\left\{X_{t}\right\}_{t \geq 0}$. We have a locally risk-free asset $\left\{B_{t}\right\}_{t \geq 0}$ with Markov-modulated dynamics

$$
d B_{t}=r_{t} B_{t} d t
$$

where $r_{t}$ takes a constant value $r_{i}$ if the economy regime variable $X_{t}$ is at the $i^{\text {th }}$ state $e_{i}$. That is,

$$
r_{t}:=\left\langle r, X_{t}\right\rangle=r_{C_{t}},
$$

where throughout $\langle\cdot, \cdot\rangle$ denotes the standard inner product in $\mathbb{R}^{N}$ and $r=\left(r_{1}, r_{2}, \ldots, r_{N}\right)^{\prime}$ is a vector of positive constants, which represent the possible risk-free short-rates in the market. The risky (default-free) asset $\left\{S_{t}\right\}_{t \geq 0}$ follows the dynamics

$$
d S_{t}=\mu_{t} S_{t} d t+\sigma_{t} S_{t} d W_{t}, \quad S_{0}=s,
$$

where $\left\{W_{t}\right\}_{t \geq 0}$ is an $\mathbb{F}$-adapted standard Brownian motion independent of $\left\{X_{t}\right\}$ under the measure $\mathbb{P}$, and

$$
\mu_{t}:=\left\langle\mu, X_{t}\right\rangle=\mu_{C_{t}}, \quad \sigma_{t}:=\left\langle\sigma, X_{t}\right\rangle=\sigma_{C_{t}}
$$

for constant vectors $\mu=\left(\mu_{1}, \mu_{2}, \ldots, \mu_{N}\right)^{\prime}$ and $\sigma=\left(\sigma_{1}, \sigma_{2}, \ldots, \sigma_{N}\right)^{\prime}$, representing the respective appreciation rates and volatilities that the risky asset can take depending on the different economic regimes.

The pricing methods we introduce in this paper can be applied to a wide range of defaultable securities such as corporate bonds, vulnerable claims, recovery payments at default, or even vulnerable call/put options on the risky stock $\left\{S_{t}\right\}_{t \geq 0}$. As a way to fix ideas, we will focus on a defaultable security, whose recovery payment at default is determined by the recovery of market value (RM) assumption (see, e.g., (McNeil et al., 2006, Section 9.4) or (Bielecki and Rutkowski, 2001, Section 8.3)). More precisely, this security delivers an $\mathcal{F}_{T}$-measurable promised payment $\mathcal{X}$ at time $T$ if no default has occured by that time (i.e. $\tau>T$ ) and, otherwise, delivers the recovery payment $\left(1-L_{\tau}\right) \gamma\left(\tau^{-} ; T\right)$ at the default time $\tau \leq T$, where $\{\gamma(t ; T)\}_{t \geq 0}$ is the pre-default value process of the security and $\left\{L_{t}\right\}_{t \geq 0}$ is an $\mathbb{F}$-adapted process, which represents the percentage loss given default. Then, the following fundamental risk-neutral valuation formula due to Duffie and Singleton (1999) (see also Proposition 8.3.3 in Bielecki and Rutkowski (2001)) is well-known:

$$
\gamma(t ; T)=\mathbb{E}^{\mathbb{Q}}\left[\mathcal{X} e^{-\int_{t}^{T}\left(r_{s}+h_{s} L_{s}\right) d s} \mid \mathcal{F}_{t}\right]
$$


where $\mathbb{Q}$ is the equivalent martingale measure used in pricing. Furthermore, denoting $\{\Gamma(t ; T)\}_{t \geq 0}$ the value of the previously described defaultable security, it follows that

$$
\Gamma(t ; T)=\mathbf{1}_{\tau>t} \gamma(t ; T)=\mathbf{1}_{\tau>t} \mathbb{E}^{\mathbb{Q}}\left[\mathcal{X} e^{-\int_{t}^{T}\left(r_{s}+h_{s} L_{s}\right) d s} \mid \mathcal{F}_{t}\right] .
$$

An important example of the defaultable security 10 is a defaultable bond given by the price process

$$
p(t, T)=\mathbf{1}_{\{\tau>t\}} \mathbb{E}^{\mathbb{Q}}\left[e^{-\int_{t}^{T}\left(r_{s}+h_{s} L_{s}\right) d s} \mid \mathcal{F}_{t}\right] .
$$

Throughout, we assume that $\mathbb{Q}$ is such that $W$ is still a standard Brownian motion and $X$ is an independent continuous-time Markov process, under $\mathbb{Q}$, with possibly different generator $A^{\mathbb{Q}}(t):=\left[a_{i, j}^{\mathbb{Q}}(t)\right]_{i, j=1,2, \ldots, N}$. The existence of the measure $\mathbb{Q}$ follows from the theory of change of measures for continuous-time Markov chains (see Section 11.2 in Bielecki and Rutkowski (2001)). Such density transformation theorems for Markov chains will play a key role in our pricing approach below. Concretely, for any $i \neq j$ and some bounded measurable functions $\kappa_{i, j}: \mathbb{R}_{+} \rightarrow(-1, \infty)$, let

$$
a_{i, j}^{\mathbb{Q}}(t):=a_{i, j}(t)\left(1+\kappa_{i, j}(t)\right),
$$

and, for $i=j$, let

$$
a_{i, i}^{\mathbb{Q}}(t):=-\sum_{k=1, k \neq i}^{N} a_{i, k}^{\mathbb{Q}}(t) .
$$

We also fix $\kappa_{i, i}(t)=0$ for $i=1, \ldots, N$. Now, consider the processes

$$
M_{t}^{i, j}:=H_{t}^{i, j}-\int_{0}^{t} a_{i, j}(u) H_{u}^{i} d u
$$

where

$$
H_{t}^{i}:=\mathbf{1}_{\left\{X_{t}=e_{i}\right\}}, \quad \text { and } \quad H_{t}^{i, j}:=\sum_{0<u \leq t} \mathbf{1}_{\left\{X_{u^{-}}=e_{i}\right\}} \mathbf{1}_{\left\{X_{u}=e_{j}\right\}}, \quad(i \neq j) .
$$

The process $\left\{M_{t}^{i, j}\right\}_{t \geq 0}$ is known to be an $\mathbb{F}$-martingale for any $i \neq j$ (see Lemma 11.2.3 in Bielecki and Rutkowski (2001)) and, since the $H$-hypothesis holds in our default framework, they are also $\mathbb{G}$-martingales. Then, we have the following result (see Proposition 11.2.3 in Bielecki and Rutkowski (2001)):

Lemma 2.1. The probability measure $\mathbb{Q}$ on $\mathbb{G}=\left(\mathcal{G}_{t}\right)_{t}$ with Radon-Nikodym density $\left\{\eta_{t}\right\}_{t \geq 0}$ given by

$$
\eta_{t}=1+\int_{(0, t]} \sum_{i, j=1}^{N} \eta_{u^{-}} \kappa_{i, j}(u) d M_{u}^{i, j}, \quad t \geq 0,
$$

is such that $X$ is a Markov process under $\mathbb{Q}$ with generator $\left[a_{i, j}^{\mathbb{Q}}(t)\right]_{i, j=1,2, \ldots, N}$.

Without loss of generality, one can take the measure $\mathbb{Q}$ in the previous lemma to be such that $W$ is still a Wiener process independent of $X$ under $\mathbb{Q}$. Analogously to (4), the process

$$
M^{\mathbb{Q}}(t):=X_{t}-X_{0}-\int_{0}^{t} A^{\mathbb{Q}}(s)^{\prime} X_{s} d s
$$

is a $\mathbb{R}^{N}$-valued martingale under $\mathbb{Q}$. Comparing $(16)$ to 4 , note that

$$
M^{\mathbb{Q}}(t)=M^{\mathbb{P}}(t)+\int_{0}^{t}\left(A(s)^{\prime}-A^{\mathbb{Q}}(s)^{\prime}\right) X_{s} d s .
$$


To be consistent with our regime-switching market model, we assume that the default intensity $h$ and loss rates $L$ are also driven by the underlying Markov process $X$; i.e.,

$$
h_{t}:=\left\langle h, X_{t}\right\rangle=h_{C_{t}}, \quad \text { and } \quad L_{t}:=\left\langle L, X_{t}\right\rangle=L_{C_{t}},
$$

for constant vectors $h=\left(h_{1}, \ldots, h_{N}\right)^{\prime}$ and $L=\left(L_{1}, \ldots, L_{N}\right)^{\prime}$, where $h_{i}>0$, and $0 \leq L_{i} \leq 1$. We emphasize that the distribution of the hazard rate process $h_{t}=\left\langle h, X_{t}\right\rangle$ under the risk-neutral measure is different from that under the historical measure, which allows to incorporate a default risk premium into our framework.

The following result states that the process $\left\{\xi_{t}^{\mathbb{P}}\right\}_{t \geq 0}$ introduced in $(2)$ is also a $\mathbb{Q}$-martingale. However, in order to indicate in the sequel when certain dynamics are being taken under $\mathbb{Q}$ or under $\mathbb{P}$, we will introduce a new notation $\left\{\xi_{t}^{\mathbb{Q}}\right\}_{t \geq 0}$. We should keep in mind throughout that $\xi^{\mathbb{Q}}=\xi^{\mathbb{P}}$. The proof of this result is presented in Appendix A.

Lemma 2.2. The process

$$
\xi_{t}^{\mathbb{Q}}:=H(t)-\int_{0}^{t}\left(1-H\left(u^{-}\right)\right) h_{u} d u
$$

is also a $(\mathbb{G}, \mathbb{Q})$ - (local) martingale.

\section{Poisson series representations for vulnerable claims}

In this section, we introduce the main tool upon which the construction of our novel algorithms as well as the semimartingales and Feynman-Kač representations of the price processes are based on. We focus on the case when the promised payoff $\mathcal{X}$ in 100 is of the form $\Xi\left(X_{T}\right)$ for a deterministic function $\Xi:\left\{e_{1}, e_{2}, \ldots, e_{N}\right\} \rightarrow \mathbb{R}$. In this case, due to the Markov property, the pre-default value process (9) can be written as

$$
\gamma(t ; T)=\Psi_{C_{t}}[\Xi](t ; T),
$$

where, for each economic regime $i \in\{1, \ldots, N\}$,

$$
\Psi_{i}[\Xi](t ; T):=\mathbb{E}^{\mathbb{Q}}\left[\Xi\left(X_{T}\right) e^{-\int_{t}^{T}\left(r_{s}+h_{s} L_{s}\right) d s} \mid X_{t}=e_{i}\right] .
$$

The key idea of our approach to compute 20 lies in changing the risk-neutral probability measure $\mathbb{Q}$ into an equivalent measure $\widetilde{\mathbb{Q}}$ such that $\left\{X_{t}\right\}_{t \leq T}$ becomes a homogeneous Markov process under $\widetilde{\mathbb{Q}}$. Such a probability measure $\widetilde{\mathbb{Q}}$ exists whenever $a_{i, j}^{\mathbb{Q}}(t)$ is strictly positive for all $t>0$ and $i \neq j$. Concretely, define functions $\tilde{\kappa}_{i, j}:[0, \infty) \rightarrow(-1, \infty)$ such that

$$
\frac{1}{N-1}=a_{i, j}^{\mathbb{Q}}(t)\left(1+\tilde{\kappa}_{i, j}(t)\right), \quad \text { for any } i \neq j, \quad \text { and } \quad \tilde{\kappa}_{i, i}=0 .
$$

We also let $\tilde{a}_{i, j}:=1 /(N-1)$ for any $i \neq j$ and $\tilde{a}_{i, i}=-1$ for any $i=1, \ldots, N$, so that $\widetilde{A}:=\left[\tilde{a}_{i, j}\right]_{i, j=1, \ldots, N}$ is a valid generator of a homogeneous Markov process with transition times determined by a homogeneous Poisson process $\left\{M_{t}\right\}_{t \geq 0}$ with intensity 1 and an embedded Markov chain $\left\{\widetilde{X}_{i}\right\}_{i \geq 1}$ with transition probabilities $p_{i, j}:=1 /(N-1)$ for $i \neq j$. Now, let us define a probability measure $\widetilde{\mathbb{Q}}$ with Radon-Nikodym density $\left\{\tilde{\eta}_{t}\right\}_{t \geq 0}$ given by

$$
\tilde{\eta}_{t}=1+\int_{(0, t]} \sum_{i, j=1}^{N} \tilde{\eta}_{u}-\tilde{\kappa}_{i, j}(u) d \widetilde{M}_{u}^{i, j},
$$

where $\widetilde{M}_{t}^{i, j}:=H_{t}^{i, j}-\int_{0}^{t} a_{i, j}^{\mathbb{Q}}(u) H_{u}^{i} d u$, and we used notation 14 . By virtue of Proposition 11.2.3 in Bielecki and Rutkowski 2001), $\left\{X_{t}\right\}_{t \geq 0}$ is a continuous Markov process with generator $\widetilde{A}$ under $\widetilde{\mathbb{Q}}$. The representation below then follows: 
Theorem 3.1. Suppose that, for any $i \neq j$, the function $a_{i, j}^{\mathbb{Q}}$ defined in 12 is such that

$$
0<\inf _{s \in[0, T]}\left|a_{i, j}^{\mathbb{Q}}(s)\right| \leq \sup _{s \in[0, T]}\left|a_{i, j}^{\mathbb{Q}}(s)\right|<\infty .
$$

Then,

$$
\Psi_{i}[\Xi](t ; T)=\mathbb{E}^{\widetilde{\mathbb{Q}}}\left[\Xi\left(X_{T}\right) e^{-\int_{t}^{T} \tilde{r}(s)^{\prime} X_{s} d s-\sum_{\left\{s \in(t, T]: \Delta X_{s} \neq 0\right\}} X_{s-}^{\prime} \widetilde{K}(s) X_{s}} \mid X_{t}=e_{i}\right],
$$

where $\widetilde{K}(t):=\left[\widetilde{K}_{i, j}(t)\right]_{i, j}$ and $\tilde{r}(t):=\left(\tilde{r}_{1}(t), \ldots, \tilde{r}_{N}(t)\right)^{\prime}$ are defined as

$$
\widetilde{K}_{i, j}(t):=-\log \left((N-1) a_{i, j}^{\mathbb{Q}}(t)\right) \mathbf{1}_{i \neq j}, \quad \tilde{r}_{i}(t):=r_{i}+h_{i} L_{i}-1-a_{i, i}^{\mathbb{Q}}(t) .
$$

As explained before, $\widetilde{\mathbb{Q}}$ has the virtue that the generator of $\left\{X_{t}\right\}_{t \geq 0}$ under $\widetilde{\mathbb{Q}}$ is given by $\tilde{a}_{i, j}=1 /(N-1)(i \neq j)$ and $\tilde{a}_{i, i}=-1$. Thus, by conditioning on the number of transitions in $(t, T]$ (which is necessarily Poisson distributed with unit intensity), we get the following series representation:

$$
\Psi_{i}[\Xi](t ; T)=\sum_{m=0}^{\infty} e^{-(T-t)} \frac{(T-t)^{m}}{m !} \Phi_{i, m}[\Xi](T-t ; T),
$$

where, denoting $\mathbb{E}_{i}^{\widetilde{\mathbb{Q}}}[\cdot]=\mathbb{E}^{\widetilde{\mathbb{Q}}}\left[\cdot \mid \widetilde{X}_{0}=e_{i}\right]$,

$$
\Phi_{i, m}[\Xi](\zeta ; T):=\mathbb{E}_{i}^{\widetilde{\mathbb{Q}}}\left[\Xi\left(\widetilde{X}_{m}\right) \exp \left\{-\sum_{n=0}^{m} \int_{\zeta U_{(n)}}^{\zeta U_{(n+1)}} \tilde{r}(T-\zeta+s)^{\prime} \widetilde{X}_{n} d s-\sum_{n=1}^{m} \widetilde{X}_{n-1}^{\prime} \widetilde{K}\left(T-\zeta+\zeta U_{(n)}\right) \widetilde{X}_{n}\right\}\right] .
$$

Here, $\left\{\widetilde{X}_{i}\right\}$ is the embedded Markov chain of $\left\{X_{t}\right\}_{t \geq 0}$ and $U_{(1)}<U_{(2)}<\cdots<U_{(m)}$ are the ordered statistics of $m$ i.i.d. uniform $[0,1]$ variables independent of $\left\{\widetilde{X}_{i}\right\}$, fixing $U_{(0)}:=0$ and $U_{(m+1)}:=11^{1}$. Thus, for instance, we have:

$$
\Phi_{i, 0}[\Xi](\zeta ; T)=\Xi\left(e_{i}\right), \quad \Phi_{i, 1}[\Xi](\zeta ; T)=\frac{\zeta^{-1}}{N-1} \sum_{j \neq i} \Xi\left(e_{j}\right) \int_{T-\zeta}^{T} e^{-\int_{0}^{v} \tilde{r}_{i}(w) d w-\int_{v}^{T} \tilde{r}_{j}(w) d w-\widetilde{K}_{i, j}(v)} d v .
$$

The series representation (25) is one of the fundamental tools in the forthcoming results. We shall apply it to find conditions for the pre-default price functions 20 to be differentiable, which in turn will lead to rigorous FeynmanKač and semimartingale representations for the pre-default price functions (9) and the vulnerable claim price process (10), respectively (see Section 4 below). Furthermore, we shall also apply the above Poisson series representation in developing our pricing methods for vulnerable contingent claims (see Section 5 below). In what follows, the dependence on $T$ will sometimes be omitted from $\Phi$ in order to lighten the notation.

\section{Semimartingale representations of vulnerable claims}

In this section, we rigorously deduce Feynman-Kač martingale representations for the pre-default price process $(20)$, and semimartingale representations for the price process of the vulnerable claim given by Eq. (10). The latter are given both under the risk-neutral probability measure $\mathbb{Q}$ and under the historical probability measure $\mathbb{P}$. The representations will be expressed in terms of the martingales (2p) and (4) (respectively, (16) and (19p). The semimartingale representations obtained here are vital in many fundamental problems of finance including hedging and portfolio optimization. Hedging with credit risky securities has attracted a lot of attention in recent years (see, e.g., Bielecki et al. (2003-a), Bielecki et al. (2003-b), Bielecki et al. (2004), and Blanchet-Scalliet and Jeanblanc (2004)), while, combined with our portfolio

\footnotetext{
${ }^{1}$ Note that, strictly speaking, the distribution of the variables $0=U_{(0)}<U_{(1)}<\cdots<U_{(m)}<U_{(m+1)}=1$ depends on $m$ and, hence, we should probably denote them as $\left\{U_{(i)}^{(m)}\right\}_{i=1}^{m}$, but for simplicity, we will omit the superscript $(m)$.
} 
optimization framework developed in Capponi and Figueroa-López $(2012)$, our results here can also be applied to portfolio optimization problems with more general vulnerable claims of the form 10 .

The representations obtained in this section are based on the following result, which in turn follows from the Poisson series representation 25) introduced in the previous section. Its proof is presented in Appendix A.

Lemma 4.1. In addition to the conditions in 22), suppose that the functions $a_{i, j}^{\mathbb{Q}}$ are continuously differentiable in $(0, T)$ and that

$$
\sup _{s \in[0, T]}\left|\frac{d a_{i, j}^{\mathbb{Q}}(s)}{d s}\right|<\infty, \quad \text { for all } i \neq j .
$$

Then, the pre-default price function $\Psi_{i}(t):=\Psi_{i}[\Xi](t ; T)$ defined in 20 is continuously differentiable for any $t \in(0, T)$.

\subsection{Markov chain dependent claims}

We proceed to establish a system of ordinary differential equations for the pre-default price functions (9) and the semimartingale representation for the price process $(10)$ in the case of a claim $\mathcal{X}$ depending only on the underlying Markov chain. An important example of a Markov chain dependent claim is the defaultable bond price, which amounts to taking $\Xi\left(e_{i}\right)=1$ for all $i=1, \ldots, N$. Similar Feynman-Kač representations have been given in Elliott and Kopp (2004), Corollary 9.8.4. However, these are given under the assumption that the price of the regime conditioned claim is a time differentiable function. Here, we fully justify the differentiability assumption using our Theorem 3.1 and Lemma 4.1 and, thus, make the analysis fully rigorous. To the best of our knowledge, the semimartingales representations are new and, as previously indicated, are crucial in, e.g., hedging and portfolio optimization problems.

The following result, whose proof is presented in Appendix $\mathrm{A}$ is the main result of this section.

Theorem 4.2. Suppose that the conditions (22) and (28) are satisfied. Then, the pre-default price function $\Psi_{i}(t):=$ $\Psi_{i}[\Xi](t ; T)$ is differentiable for any $t \in(0, T)$. Furthermore, the following statements hold:

(1) The vector of pre-default prices $\Psi(t)=\left(\Psi_{1}(t), \ldots, \Psi_{N}(t)\right)^{\prime}$ satisfies the following coupled system of backward Ordinary Differential Equations (ODE):

$$
\begin{aligned}
\frac{d \Psi_{i}(t)}{d t} & =\left(r_{i}+h_{i} L_{i}-a_{i, i}^{\mathbb{Q}}(t)\right) \Psi_{i}(t)-\sum_{j \neq i} a_{i, j}^{\mathbb{Q}}(t) \Psi_{j}(t), \quad 0<t<T, \\
\Psi_{i}(T) & =\Xi\left(e_{i}\right), \quad i=1, \ldots, N .
\end{aligned}
$$

(2) The prices process 10$)$ of the vulnerable claim with payoff $\Xi\left(X_{T}\right)$ has the following semimartingale representation:

$$
d \Gamma(t, T)=\Gamma\left(t^{-} ; T\right)\left(\left[r_{t}+h_{t}\left(L_{t}-1\right)\right] d t+\frac{\left\langle\Psi(t), d M^{\mathbb{Q}}(t)\right\rangle}{\left\langle\Psi(t), X_{t^{-}}\right\rangle}-d \xi_{t}^{\mathbb{Q}}\right), \quad 0 \leq t<T, \quad t<\tau,
$$

under the risk-neutral probability measure $\mathbb{Q}$.

(3) The prices process (10) of the vulnerable claim with payoff $\Xi\left(X_{T}\right)$ has the following semimartingale representation:

$$
d \Gamma(t, T)=\Gamma\left(t^{-}, T\right)\left(\left[r_{t}+h_{t}\left(L_{t}-1\right)+D(t)\right] d t+\frac{\left\langle\Psi(t), d M^{\mathbb{P}}(t)\right\rangle}{\left\langle\Psi(t), X_{t^{-}}\right\rangle}-d \xi_{t}^{\mathbb{P}}\right), \quad 0 \leq t<T, \quad t<\tau,
$$

under the historical probability measure $\mathbb{P}$, where $D(t):=\left\langle\left(D_{1}(t), \ldots, D_{N}(t)\right)^{\prime}, X_{t}\right\rangle$ with

$$
D_{i}(t):=\sum_{j=1}^{N}\left(a_{i, j}(t)-a_{i, j}^{\mathbb{Q}}(t)\right) \frac{\Psi_{j}(t)}{\Psi_{i}(t)}=\sum_{j \neq i}\left(a_{i, j}(t)-a_{i, j}^{\mathbb{Q}}(t)\right)\left(\frac{\Psi_{j}(t)}{\Psi_{i}(t)}-1\right) .
$$


Remark 4.3. As a direct consequence of the previous result, in the time-invariant case (i.e., $\left.a_{i, j}^{\mathbb{Q}}(t) \equiv a_{i, j}^{\mathbb{Q}}\right)$, the solution of (29) can be expressed in "closed-form" as

$$
\psi(t ; T)=e^{-(T-t) F_{\psi}} \boldsymbol{\Xi},
$$

where $\boldsymbol{\Xi}=\left(\Xi\left(e_{1}\right), \ldots, \Xi\left(e_{N}\right)\right)^{\prime}$ and the components of the matrix $F_{\psi}$ are

$$
\left[F_{\psi}\right]_{i, i}=r_{i}+h_{i} L_{i}-a_{i, i}^{\mathbb{Q}}, \quad \text { and } \quad\left[F_{\psi}\right]_{i, j}=-a_{i, j}^{\mathbb{Q}}, \quad i \neq j .
$$

\subsection{Markov chain and diffusion dependent claims}

In this section, we provide the Feynman-Kač type partial differential equations and the semimartingale representations of the price process for claims whose payoffs depend both on the Brownian paths as well as on the underlying Markov chain. For non-vulnerable claims, Elliott et al. (2007) and Yao et al. (2006) obtained similar Feynman-Kač representations. Here, the analysis is extended to deal with vulnerable claims.

We follow Elliott et al. (2005) and choose the the risk-neutral measure $\mathbb{Q}$ such that

$$
\left.\frac{d \mathbb{Q}}{d \mathbb{P}}\right|_{\mathcal{F}_{t}}=\exp \left\{\int_{0}^{t}\left(\frac{r_{s}-\mu_{s}}{\sigma_{s}}\right) d W_{s}-\frac{1}{2} \int_{0}^{t}\left(\frac{r_{s}-\mu_{s}}{\sigma_{s}}\right)^{2} d s\right\} .
$$

In particular, the $\left(\mathcal{F}_{t}, \mathbb{Q}\right)$ Brownian motion is given by

$$
W_{t}^{\mathbb{Q}}=W_{t}+\int_{0}^{t}\left(\frac{r_{s}-\mu_{s}}{\sigma_{s}}\right) d s
$$

Note that, without loss of generality, we can take $\mathbb{Q}$ so that under it, $\left\{X_{t}\right\}_{t \geq 0}$ is still a continuous-time Markov process with generator $A^{\mathbb{Q}}$, independent of $\left\{W_{t}^{\mathbb{Q}}\right\}_{t \geq 0}$.

Next, we consider a pre-default price process of the form $(9)$, where $\mathcal{X}=\varrho\left(S_{T}\right)$, for a deterministic function $\varrho$. We denote it by $\Pi(t ; s)=\left(\Pi_{1}(t ; s), \ldots, \Pi_{N}(t ; s)\right)^{\prime}$, where

$$
\Pi_{i}(t ; s):=\mathbb{E}^{\mathbb{Q}}\left[\varrho\left(S_{T}\right) e^{-\int_{t}^{T}\left(r_{s}+h_{s} L_{s}\right) d s} \mid X_{t}=e_{i}, S_{t}=s\right] .
$$

The proof is presented in Appendix section.

Theorem 4.4. Assume that each $\Pi_{i}(t ; s)$ is continuously differentiable in $t \in(0, T)$ and twice continuously differentiable in $s \in(0, \infty)$. Then, the following statements hold:

(1) The vector of pre-default prices $\Pi(t ; s)=\left(\Pi_{1}(t ; s), \ldots, \Pi_{N}(t ; s)\right)^{\prime}$ satisfies the following coupled system of backward Partial Differential Equations (PDE), in the domain $0 \leq t<T, 0<s<\infty$ :

$$
\begin{aligned}
& \frac{\partial \Pi_{i}(t ; s)}{\partial t}+r_{i}\left(s \frac{\partial \Pi_{i}(t ; s)}{\partial s}-\Pi_{i}(t ; s)\right)-\left(h_{i} L_{i}-a_{i, i}^{\mathbb{Q}}(t)\right) \Pi_{i}(t ; s)+\frac{1}{2} \sigma_{i}^{2} s^{2} \frac{\partial^{2} \Pi_{i}(t ; s)}{\partial^{2} s}+\sum_{j \neq i} a_{i, j}^{\mathbb{Q}}(t) \Pi_{j}(t ; s)=0 \\
& \gamma_{i}(T, s)=\varrho(s), \quad i=1, \ldots, N .
\end{aligned}
$$

(2) The prices process $(10)$, denoted hereafter by $\Gamma\left(t, T ; S_{t}\right)$, of the vulnerable claim with payoff $\mathcal{X}=\varrho\left(S_{T}\right)$ has the following semimartingale representation, for $0 \leq t<T, 0<S_{t}<\infty, t<\tau$ :

$$
d \Gamma\left(t, T ; S_{t}\right)=\Gamma\left(t^{-}, T ; S_{t-}\right)\left(\left[r_{t}+h_{t}\left(L_{t}-1\right)\right] d t+\frac{\left\langle\Pi\left(t ; S_{t}\right), d M^{\mathbb{Q}}(t)\right\rangle}{\left\langle\Pi\left(t ; S_{t}\right), X_{t^{-}}\right\rangle}+\sigma_{t} S_{t} \frac{\frac{\partial\left\langle\Pi\left(t ; S_{t}\right), X_{t}\right\rangle}{\partial s}}{\left\langle\Pi\left(t ; S_{t}\right), X_{t^{-}}\right\rangle} d W_{t}^{\mathbb{Q}}-d \xi_{t}^{\mathbb{Q}}\right)
$$

under the risk-neutral probability measure $\mathbb{Q}$. 
(3) The prices process (10) of the vulnerable claim with payoff $\varrho\left(S_{T}\right)$ has the following semimartingale representation, for $0 \leq t<T, 0<S_{t}<\infty, t<\tau$ :

$$
\begin{gathered}
d \Gamma\left(t, T ; S_{t}\right)=\Gamma\left(t-, T ; S_{t-}\right)\left(\left[r_{t}+h_{t}\left(L_{t}-1\right)+D\left(t, S_{t}\right)+\frac{S_{t} \frac{\partial\left\langle\Pi\left(t ; S_{t}\right), X_{t}\right\rangle}{\partial s}}{\left\langle\Pi\left(t ; S_{t}\right), X_{t^{-}}\right\rangle}\left(\mu_{t}-r_{t}\right)\right] d t\right. \\
\left.+\frac{\left\langle\Pi\left(t ; S_{t}\right), d M^{\mathbb{P}}(t)\right\rangle}{\left\langle\Pi\left(t ; S_{t}\right), X_{t^{-}}\right\rangle}+\sigma_{t} S_{t} \frac{\frac{\partial\left\langle\Pi\left(t ; S_{t}\right), X_{t}\right\rangle}{\partial s}}{\left\langle\Pi\left(t ; S_{t}\right), X_{t^{-}}\right\rangle} d W_{t}-d \xi_{t}^{\mathbb{P}}\right),
\end{gathered}
$$

under the historical probability measure $\mathbb{P}$, where $D\left(t, S_{t}\right):=\left\langle\left(D_{1}\left(t, S_{t}\right), \ldots, D_{N}\left(t, S_{t}\right)\right)^{\prime}, X_{t}\right\rangle$ with

$$
D_{i}\left(t, S_{t}\right):=\sum_{j=1}^{N}\left(a_{i, j}(t)-a_{i, j}^{\mathbb{Q}}(t)\right) \frac{\Pi_{j}\left(t ; S_{t}\right)}{\Pi_{i}\left(t ; S_{t}\right)}=\sum_{j \neq i}\left(a_{i, j}(t)-a_{i, j}^{\mathbb{Q}}(t)\right)\left(\frac{\Pi_{j}\left(t ; S_{t}\right)}{\Pi_{i}\left(t ; S_{t}\right)}-1\right) .
$$

\section{$5 \quad$ Novel algorithm for pricing vulnerable claims}

This section introduces a novel algorithm for pricing vulnerable claims. Concretely, Section 5.1 presents the basic algorithm and analyzes its computational complexity. Section 5.2 develops an error analysis of the algorithm by providing precise bounds for approximation error of the method. Section 5.3 then builds on the basic algorithm and extends it to the pricing self-decomposable claims on the underlying chain. Section 5.4 introduces a novel algorithm to price vulnerable European type options using the basic algorithm.

\subsection{Markov chain dependent claims}

In this section, we develop a novel efficient algorithm for pricing a claim whose payoff depends on the underlying economic regime in place $\left\{X_{t}\right\}_{t \geq 0}$. This algorithm is used to price simple European claims of the form $\Xi\left(X_{T}\right)$ for a deterministic function $\Xi$ as in Section 3 . Let us consider the pre-default price function 20 under the $i^{\text {th }}$ regime. Our approach to compute 20 builds on the series representation $\left[25\right.$. Concretely, we will derive a formula for $\Phi_{i, m}[\Xi](\zeta)$ when the risk-neutral generator $A^{\mathbb{Q}}$ is time-invariant. The formula will be expressed in terms of the Laplace transform of the "symmetric" Dirichlet distribution, defined by

$$
\mathcal{L}_{m}\left(x_{1}, \ldots, x_{m}\right):=m ! \int_{T_{m}} e^{-\sum_{j=1}^{m} x_{j} \lambda_{j}} d \lambda
$$

where $T_{m}:=\left\{\left(\lambda_{1}, \ldots, \lambda_{m}\right) \in \mathbb{R}^{m}: \lambda_{i} \geq 0, \sum_{i=1}^{m} \lambda_{i} \leq 1\right\}$. The proof of the following result is given in Appendix A

Proposition 5.1. Suppose that $a_{i, j}^{\mathbb{Q}}(t) \equiv a_{i, j}^{\mathbb{Q}}$ (hence, $\widetilde{K}$ and $\widetilde{r}$ are also time-invariant). Then, for $m \geq 1$, we have that

$$
\Phi_{i, m}[\Xi](\zeta)=\frac{1}{(N-1)^{m}} \sum_{\left(\tilde{e}_{1}, \ldots, \tilde{e}_{m}\right)} \Xi\left(\tilde{e}_{m}\right) e^{-\zeta \tilde{r}^{\prime} \tilde{e}_{m}-\sum_{n=1}^{m} \tilde{e}_{n-1}^{\prime} \widetilde{K} \tilde{e}_{n}} \mathcal{L}_{m}\left(\zeta \tilde{r}^{\prime}\left(\tilde{e}_{0}-\tilde{e}_{m}\right), \ldots, \zeta \tilde{r}^{\prime}\left(\tilde{e}_{m-1}-\tilde{e}_{m}\right)\right),
$$

where we set $\tilde{e}_{0}=e_{i}$ and the above summation is over all "paths" $\left(\tilde{e}_{1}, \ldots, \tilde{e}_{m}\right)$ such that $\tilde{e}_{j} \in\left\{e_{1}, \ldots, e_{N}\right\}$ and $\tilde{e}_{j} \neq \tilde{e}_{j-1}$, for $j=1, \ldots, m$.

Remark 5.2. Note that

$$
\Phi_{i, 0}[\Xi](\zeta)=\Xi\left(e_{i}\right) e^{-\zeta \tilde{r}_{i}}, \quad \Phi_{i, 1}[\Xi](\zeta)=\frac{1}{(N-1)} \sum_{j \in\{1, \ldots, N\} \backslash\{i\}} \Xi\left(e_{j}\right) e^{-\zeta \tilde{r}_{j}-\widetilde{K}_{i, j}} \frac{1}{\zeta\left(\tilde{r}_{i}-\tilde{r}_{j}\right)}\left(1-e^{-\zeta\left(\tilde{r}_{i}-\tilde{r}_{j}\right)}\right) .
$$

For a general $m$, the following Taylor approximation around the origin will turn out to be quite useful to compute the Dirichlet Laplace transform 40):

$$
\mathcal{L}_{m}\left(x_{1}, \ldots, x_{m}\right)=1-\frac{\sum_{i=1}^{m} x_{i}}{m+1}+\frac{\sum_{i=1}^{m} x_{i}^{2}+\frac{1}{2} \sum_{i \neq j} x_{i} x_{j}}{(m+1)(m+2)}+O\left(\left\|\left(x_{1}, \ldots, x_{m}\right)\right\|^{3 / 2}\right) .
$$


In order to evaluate the option price, the infinite series 25 will be truncated and the Laplace transform in (41) may also be approximated by (43). Both of these approximations are valid when time-to-maturity $\zeta=T-t$ is small. Hence, it useful to express the option price 23 in terms of the price of options with shorter expiration. Concretely, fix a small mesh parameter $\delta:=T / k$ for a given positive integer $k$ and let

$$
I_{u, v}:=-\int_{u}^{v} \tilde{r}^{\prime} X_{s} d s-\sum_{s \in(u, v]: \Delta X_{s} \neq 0} X_{s^{-}}^{\prime} \tilde{K} X_{s} .
$$

Then, using the tower and Markov properties, the time 0 price of the vulnerable claim may be computed as follows

$$
\begin{aligned}
\Psi_{i}[\Xi](0 ; T) & =\mathbb{E}^{\widetilde{\mathbb{Q}}}\left[e^{I_{0, T}} \Xi\left(X_{T}\right) \mid X_{0}=e_{i}\right]=\mathbb{E}^{\widetilde{\mathbb{Q}}}\left[e^{I_{0, \delta}} \mathbb{E}^{\widetilde{\mathbb{Q}}}\left[\Xi\left(X_{T}\right) e^{I_{\delta, T}} \mid \mathcal{F}_{\delta}\right] \mid X_{0}=e_{i}\right] \\
& =: \mathbb{E}^{\widetilde{\mathbb{Q}}}\left[e^{I_{0, \delta}} \widetilde{\Xi}\left(X_{\delta}\right) \mid X_{0}=e_{i}\right]=\Psi_{i}[\widetilde{\Xi}](0 ; \delta),
\end{aligned}
$$

where we introduced a new payoff function $\widetilde{\Xi}$ defined as $\widetilde{\Xi}\left(e_{j}\right)=\Psi_{j}[\Xi](0 ; T-\delta)$. Note that the last expression in (45) represents the price of an option with short maturity $\delta$ and, hence, it can be accurately computed by taking $M$ terms in 25 and (possibly) using 43 . In order to evaluate the option's payoff $\widetilde{\Xi}$, we apply again the procedure 45, replacing $T$ by $T-\delta$. Computationally, one can create a recursive or iterative implementation in order to evaluate (45). The pseudo-code of the proposed iterative algorithm is given in Appendix B (Algorithm 1 therein).

Remark 5.3. The previous method can be adapted to deal with sufficiently smooth time dependent functions $\tilde{r}(t)$ and $\tilde{K}(t)$ (e.g., continuously differentiable in $[0, T]$ ). Indeed, as in (45) and using notation (5), we have

$$
\Psi_{i}[\Xi](t ; T)=\mathbb{E}^{\widetilde{\mathbb{Q}}}\left[e^{I_{t, T}} \Xi\left(X_{T}\right) \mid X_{t}=e_{i}\right]=\mathbb{E}^{\widetilde{\mathbb{Q}}}\left[e^{I_{t, t+\delta}} \Psi_{C_{t+\delta}}[\Xi](t+\delta ; T) \mid X_{t}=e_{i}\right]=\Psi_{i}[\widetilde{\Xi}](t ; t+\delta),
$$

fixing $\widetilde{\Xi}\left(e_{i}\right):=\Psi_{i}[\Xi](t+\delta ; T)$. Then, for sufficiently small $\delta, \Psi_{i}[\Xi](t ; T)$ can be accurately computed as if $\tilde{r}$ and $\tilde{K}$ were time-invariant during the period $[t, t+\delta]$. We analyze the error of this approach in the section 5.2.2 below (see also the end of Section 5.2.1 for another approach).

Remark 5.4. The complexity of the proposed method (Algorithm 1 in Appendix B) can be evaluated as follows. Let

$$
\widetilde{\Psi}_{i}^{(M)}[\Xi](t ; T):=\sum_{m=0}^{M-1} e^{-(T-t)} \frac{(T-t)^{m}}{m !} \Phi_{i, m}[\Xi](T-t) .
$$

The computational complexity to evaluate $\widetilde{\Psi}_{i}^{(M)}[\Xi](t ; T)$ for each $i$ is $O\left(N^{M-1}\right)$. Indeed, for each $m$, it is required to evaluate $\Phi_{i, m}[\Xi](T-t)$. This, in turn, requires at most $N^{M-1}$ evaluations of the Laplace transform of the symmetric Dirichlet distribution (one for each path $\left(\tilde{e}_{0}, \ldots, \tilde{e}_{m}\right)$ such that $\tilde{e}_{0}=e_{i}$ and $\tilde{e}_{j} \neq \tilde{e}_{j-1}$ for $j=1, \ldots, m$ as seen in (41)). Therefore, the total computational complexity to evaluate (47) is $O\left(N^{M-1}\right)$ and, thus, the algorithm has complexity $O\left(N^{M}\right)$ to compute the prices conditional on all starting regimes. We also remark that, for a fixed $M$, the computation of $\Psi_{i}[\Xi](0 ; T)$ can be sped up greatly by saving the paths $\left(\tilde{e}_{1}, \ldots, \tilde{e}_{m}\right)$ needed for 41) at the beginning and reusing them for each evaluation of $\Phi_{i, m}[\Xi](\zeta)$ with $\zeta \in\{\delta, \ldots, k \delta\}$.

\subsection{Error analysis}

We now proceed to give precise error bounds for our proposed method. First, we need to extend the framework of our algorithm. In this section we fix positive integers $M$ and $k$, and an integer $r \in\{1, \ldots, M\}$. We also let $\delta=T / k$ and $\mathbf{1}\left(e_{i}\right)=1$, for all $i=1, \ldots, N$. Suppose the following conditions hold true:

(1) The functionals

$$
\Phi_{i, 0}[\Xi](u ; v), \ldots, \Phi_{i, r-1}[\Xi](u ; v)
$$

defined in (26) can be computed exactly for any payoff function $\Xi:\left\{e_{1}, \ldots, e_{N}\right\} \rightarrow \mathbb{R}$ and any $0 \leq u \leq v \leq T$. 
(2) For any $m \in\{r, \ldots, M-1\}, i \in\{1, \ldots, N\}, \Xi:\left\{e_{1}, \ldots, e_{N}\right\} \rightarrow \mathbb{R}$, and $0 \leq \delta \leq T$, there is an approximation $\widetilde{\Phi}_{i, m}[\Xi](\delta ; T)$ of $\Phi_{i, m}[\Xi](\delta ; T)$, linear in $\Xi$, such that

$$
\begin{aligned}
& \left|\widetilde{\Phi}_{i, m}[\Xi](\delta ; T)-\Phi_{i, m}[\Xi](\delta ; T)\right| \leq B \max _{\ell}\left|\Xi\left(e_{\ell}\right)\right| \delta^{j}, \quad \text { for all } \delta<\delta_{0}, \\
& \left|\widetilde{\Phi}_{i, m}[\Xi](\delta ; T)\right| \leq \Phi_{i, m}[\mathbf{1}](\delta ; T) \max _{\ell}\left|\Xi\left(e_{\ell}\right)\right|, \quad \text { for all } \delta<\delta_{0},
\end{aligned}
$$

for some $0<\delta_{0} \leq T$ and $j \geq 1$ and a constant $B<\infty$, independent of $\delta, i, m$, and $\Xi$.

Next, define the following approximation for the pre-default payoff function $\Psi_{i}[\Xi](t ; T)$ :

$$
\widetilde{\Psi}_{i}^{(M, r)}[\Xi](t ; T)=\sum_{m=0}^{r-1} e^{-(T-t)} \frac{(T-t)^{m}}{m !} \Phi_{i, m}[\Xi](T-t ; T)+\sum_{m=r}^{M-1} e^{-(T-t)} \frac{(T-t)^{m}}{m !} \widetilde{\Phi}_{i, m}[\Xi](T-t ; T) .
$$

Note that the case $r=M$ essentially amounts to approximating the infinite series approximation 25 by the summation of the first $M$ terms and, hence, that the $M$ terms $\Phi_{i, 0}[\Xi](u ; v), \ldots, \Phi_{i, M-1}[\Xi](u ; v)$ are computed exactly.

Consider the following generalized algorithm to approximate $\Psi[\Xi](0 ; T):=\left(\Psi_{1}[\Xi](0 ; T), \ldots, \Psi_{N}[\Xi](0 ; T)\right)^{\prime}$, which extends our approach in Section 5.1

(A) Approximate $\Psi_{i}[\Xi]((k-1) \delta ; T)$ by the functional $\widetilde{\Psi}_{i}^{(M, r)}[\Xi]((k-1) \delta ; T)$ in $[50)$; denote this first step approximation $\widetilde{\Psi}^{(M, r, k)}[\Xi]((k-1) \delta ; T)$;

(B) For each $j$ from $k-2$ to 0 (going backwards), compute iteratively

$$
\widetilde{\Psi}^{(M, r, k)}[\Xi](j \delta ; T)=\widetilde{\Psi}^{(M, r)}\left[\widetilde{\Xi}^{(j)}\right](j \delta ;(j+1) \delta),
$$

where $\widetilde{\Xi}^{(j)}$ is computed as

$$
\widetilde{\Xi}^{(j)}\left(e_{i}\right):=\widetilde{\Psi}_{i}^{(M, r, k)}[\Xi]((j+1) \delta ; T) .
$$

The following result will be important in the subsequent error analysis. Its proof is given in the Appendix A.

Theorem 5.5. Under the above conditions (1)-(2), the previous algorithm (A)-(B) will result in a price approximation $\widetilde{\Psi}_{i}^{(M, r, k)}[\Xi](0 ; T)$ such that

$$
\max _{i \in\{1, \ldots, N\}}\left|\Psi_{i}[\Xi](0 ; T)-\widetilde{\Psi}_{i}^{(M, r, k)}[\Xi](0 ; T)\right| \leq D \frac{T^{\alpha}}{k^{\alpha-1}},
$$

where $\alpha:=(r+j) \wedge M$ and $D=\|\Xi\|_{\infty}(1+B)$ with $B$ defined as in (48) and $\|\Xi\|_{\infty}:=\max _{\ell=1, \ldots, N}\left|\Xi\left(e_{\ell}\right)\right|$.

The following result relaxes the condition (49), but does not yield a bound as explicit as in $(52)$.

Corollary 5.6. Suppose that the above conditions (1)-(2) hold true, but replacing (49) with the following milder condition:

$$
\left|\widetilde{\Phi}_{i, m}[\Xi](\delta ; T)\right| \leq C \max _{\ell}\left|\Xi\left(e_{\ell}\right)\right|, \quad \text { for all } \delta<\delta_{0},
$$

for some $0<\delta_{0} \leq T$ and a constant $C<\infty$, independent of $\delta, i, m$, and $\Xi$. Then, there exists a constant $E$ such that, for all $k \geq 1$,

$$
\max _{i \in\{1, \ldots, N\}}\left|\Psi_{i}[\Xi](0 ; T)-\widetilde{\Psi}_{i}^{(M, r, k))}[\Xi](0 ; T)\right| \leq E \frac{T^{\alpha}}{k^{\alpha-1}},
$$

where $\alpha:=(r+j) \wedge M$.

We now consider different applications of our previous general approximation framework. 


\subsubsection{Error analysis when the $\Phi_{i, m}$ 's are exact}

As a direct consequence of Theorem 5.5 by taking $r=M$ and $B=0$, the approximation

$$
\widetilde{\Psi}_{i}^{(M)}[\Xi](t ; T):=\sum_{m=0}^{M-1} e^{-(T-t)} \frac{(T-t)^{m}}{m !} \Phi_{i, m}[\Xi](T-t),
$$

attains the following error:

$$
\max _{i \in\{1, \ldots, N\}}\left|\Psi_{i}[\Xi](0 ; T)-\widetilde{\Psi}_{i}^{(M)}[\Xi](t ; T)\right| \leq\|\Xi\|_{\infty} \times \frac{T^{M}}{k^{M-1}} .
$$

As indicated in the Remark 5.2 , for time-invariant generators, $\Phi_{i, 0}[\Xi]$ and $\Phi_{i, 1}[\Xi]$ can be computed in closed form. Hence, using only these two values, the bond prices can be evaluated up to an error of order $T^{2} / k$ using $k$ iterations and with a maximal polynomial complexity of $O\left(N^{3}\right)$. Furthermore, even in the case of time-dependent generators, the functionals $\Phi_{i, 0}[\Xi](\zeta ; T)$ and $\Phi_{i, 1}[\Xi](\zeta ; T)$ can be computed by the expressions (27) (at the cost of computing a single integral) and, thus, the same error will hold true. This last observation partly justifies our claim in Remark 5.3 .

\subsubsection{Error analysis for smooth time-dependent generators}

In the case of time-dependent generators, a natural approach is to approximate $\Phi_{i, m}[\Xi](\delta ; T)$ when $\delta$ is small by freezing the values of $\tilde{r}$ and $\tilde{K}$ at the beginning of the integration interval $[T-\delta, T]$. Concretely, consider the approximation

$$
\widetilde{\Phi}_{i, m}[\Xi](\delta ; T):=\mathbb{E}_{i}^{\widetilde{\mathbb{Q}}}\left[\Xi\left(\widetilde{X}_{m}\right) \exp \left\{-\sum_{n=0}^{m} \int_{\delta U_{(n)}}^{\delta U_{(n+1)}} \tilde{r}(T-\delta)^{\prime} \widetilde{X}_{n} d s-\sum_{n=1}^{m} \widetilde{X}_{n-1}^{\prime} \widetilde{K}(T-\delta) \widetilde{X}_{n}\right\}\right]
$$

These approximations can be expressed in closed form in terms of "symmetric" Dirichlet Laplace transforms as in Proposition 5.1. Obviously, (55) satisfies (53). In order to verify the validity of (48), let us assume that for all $p, q$, the functions $\tilde{r}_{p}$ and $\widetilde{K}_{p, q}$ are continuously differentiable on $[0, T]$. Denoting the random variable inside the expectation on the right-hand side of $(55)$ by $G$ and from the definitions $(26)$ and (55), note that

$$
\begin{aligned}
& \left|\widetilde{\Phi}_{i, m}[\Xi](\delta ; T)-\Phi_{i, m}[\Xi](\delta ; T)\right| \\
& \quad \leq \mathbb{E}_{i}^{\widetilde{\mathbb{Q}}}\left[G\left|1-e^{-\sum_{n=0}^{m} \int_{\delta U_{(n)} U_{(n+1)}}\left[\tilde{r}(T-\delta+s)^{\prime}-\tilde{r}(T-\delta)^{\prime}\right] \widetilde{X}_{n} d s-\sum_{n=1}^{m} \widetilde{X}_{n-1}^{\prime}\left[\widetilde{K}\left(T-\delta+\delta U_{(n)}\right)-\widetilde{K}(T-\delta)\right] \widetilde{X}_{n}}\right|\right] \\
& \quad \leq \mathbb{E}_{i}^{\widetilde{\mathbb{Q}}}\left[G\left|1-e^{-\delta^{2} \max _{p} \sup _{s \in[T-\delta, T]}\left|\tilde{r}_{p}^{\prime}(s)\right|-m \delta \max _{p \neq q} \sup _{s \in[T-\delta, T]}\left|\widetilde{K}_{p, q}^{\prime}(s)\right|}\right|\right] \\
& \leq B\|\Xi\|_{\infty} \delta,
\end{aligned}
$$

for some constant $B$ independent of $\Xi$. Thus, 48 is satisfied with $j=1$. In particular, if we use the approximation (55) for $m=1, \ldots, M-1$ (so that $r=1$ ), then the parameter $\alpha$ of Theorem 5.5 will be two and the resulting pre-default claim price approximation will attain an error bound of the form:

$$
\max _{i \in\{1, \ldots, N\}}\left|\Psi_{i}[\Xi](0 ; T)-\widetilde{\Psi}_{i}^{(M)}[\Xi](t ; T)\right| \leq E \frac{T^{2}}{k} .
$$

\subsubsection{Error analysis arising from the Taylor approximations}

Another natural issue is to quantify and control the error arising from using a Taylor approximation to the Dirichlet Laplace transforms 40 as suggested in Remark 5.2. Concretely, let us recall the following expression from Proposition 5.1

$$
\Phi_{i, m}[\Xi](\zeta)=\frac{1}{(N-1)^{m}} \sum_{\substack{\left(\tilde{e}_{1}, \ldots, \tilde{e}_{m}\right) \\ \tilde{e}_{i} \neq \tilde{e}_{i+1}}} \Xi\left(\tilde{e}_{m}\right) e^{-\zeta \tilde{r}^{\prime} \tilde{e}_{m}-\sum_{n=1}^{m} \tilde{e}_{n-1}^{\prime} \tilde{K} \tilde{e}_{n}} \mathcal{L}_{m}\left(\zeta \tilde{r}^{\prime}\left(\tilde{e}_{0}-\tilde{e}_{m}\right), \ldots, \zeta \tilde{r}^{\prime}\left(\tilde{e}_{m-1}-\tilde{e}_{m}\right)\right)
$$


valid for time-invariant generators. Next, we consider the following "Taylor approximation":

$$
\widetilde{\Phi}_{i, m}[\Xi](\zeta)=\frac{1}{(N-1)^{m}} \sum_{\substack{\left(\tilde{e}_{1}, \ldots, \tilde{e}_{m}\right) \\ \tilde{e}_{i} \neq \tilde{e}_{i+1}}} \Xi\left(\tilde{e}_{m}\right) e^{-\zeta \tilde{r}^{\prime} \tilde{e}_{m}-\sum_{n=1}^{m} \tilde{e}_{n-1}^{\prime} \tilde{K}_{e} \tilde{e}_{n}} \widehat{\mathcal{L}}_{m}\left(\zeta \tilde{r}^{\prime}\left(\tilde{e}_{0}-\tilde{e}_{m}\right), \ldots, \zeta \tilde{r}^{\prime}\left(\tilde{e}_{m-1}-\tilde{e}_{m}\right)\right),
$$

where we approximate the Laplace transform 40 by the following Taylor polynomial of $p^{\text {th }}$-order:

$$
\widehat{\mathcal{L}}_{m}\left(x_{1}, \ldots, x_{m}\right):=\widehat{\mathcal{L}}_{m}^{(p)}\left(x_{1}, \ldots, x_{m}\right):=m ! \sum_{\ell=0}^{p} \int_{T_{m}} \frac{\langle\mathbf{x}, \lambda\rangle^{\ell}}{\ell !} d \lambda .
$$

Let us now verify the conditions for Corollary 5.6. It is easy to verify that $\widetilde{\Phi}_{i, m}[\Xi]$ satisfies the condition $[53$. It remains to show the validity of [48. First note that, by the Cauchy-Schwarz's and Jensen's inequalities,

$$
\begin{aligned}
\left|\mathcal{L}_{m}\left(x_{1}, \ldots, x_{m}\right)-\widehat{\mathcal{L}}_{m}^{(p)}\left(x_{1}, \ldots, x_{m}\right)\right| & \leq m ! \sum_{i=p+1}^{\infty} \int_{T_{m}} \frac{|\langle\mathbf{x}, \lambda\rangle|^{i}}{i !} d \lambda \leq m ! \sum_{i=p+1}^{\infty} \frac{\|x\|^{i}}{i !} \int_{T_{m}}\|\lambda\|^{i} d \lambda \\
& \leq m ! \sum_{i=p+1}^{\infty} m^{i / 2-1} \frac{\|x\|^{i}}{i !} \int_{T_{m}}\left(\sum_{\ell=1}^{m} \lambda_{\ell}^{i}\right) d \lambda \\
& \leq \sum_{i=p+1}^{\infty} m^{i / 2} \frac{\|x\|^{i}}{i !} \frac{m ! i !}{(m+i) !}
\end{aligned}
$$

where we had also used the fact that $m ! \int_{T_{m}} \lambda_{\ell}^{i} d \lambda$ coincides with $i^{\text {th }}$ moment of a $\operatorname{Beta}(1, m)$ random variable, which is known to be $m ! i ! /(m+i)$ !. Using the previous estimate, it follows that

$$
\left|\widetilde{\Phi}_{i, m}[\Xi](\zeta)-\Phi_{i, m}[\Xi](\zeta)\right| \leq K \frac{\zeta^{p+1}}{(N-1)^{m}} \sum_{\substack{\left(\tilde{e}_{1}, \ldots, \tilde{e}_{m}\right) \\ \tilde{e}_{i} \neq \tilde{e}_{i+1}}} \Xi\left(\tilde{e}_{m}\right) e^{-\zeta \tilde{r}^{\prime} \tilde{e}_{m}-\sum_{n=1}^{m} \tilde{e}_{n-1}^{\prime} \tilde{K} \tilde{e}_{n}}=: K \zeta^{p+1} \widetilde{C}_{m}
$$

for an absolute constant $K$. Finally, its is clear that

$$
\widetilde{C}_{m}=\frac{1}{(N-1)^{m}} \sum_{\substack{\left(\tilde{e}_{1}, \ldots, \tilde{e}_{m}\right) \\ \tilde{e}_{i} \neq \tilde{e}_{i+1}}} \Xi\left(\tilde{e}_{m}\right) e^{-\zeta \tilde{r} \tilde{e}_{m}-\sum_{n=1}^{m} \tilde{e}_{n-1}^{\prime} \tilde{K} \tilde{e}_{n}}
$$

can be bounded by $\|\Xi\|_{\infty} R$, for some constant $R$. In light of [58, Corollary 5.6 implies that the previous Taylor-based approximations will attain an error of the form

$$
\max _{i \in\{1, \ldots, N\}}\left|\Psi_{i}[\Xi](0 ; T)-\widetilde{\Psi}_{i}^{(M, r, k))}[\Xi](0 ; T)\right| \leq E \frac{T^{\alpha}}{k^{\alpha-1}},
$$

with $\alpha:=r+(p+1)=(3+p) \wedge M$ if we take $M>2$ and use the exact values 42$\}$ for $m \in\{0,1\}$ and Taylor approximations of $p^{\text {th }}$ order for $m=2, \ldots, M$ (so that $r=2$ ).

\subsection{Pricing of decomposable claims on the underlying Markov process}

We proceed to extend our approach above to price a class of path-dependent claims, termed self-decomposable claims, whose payoffs can be decomposed into shorter maturity payoffs. We illustrate our method for a type of barrier option on the Markov process $\left\{X_{t}\right\}_{t \geq 0}$. As it is evident from [45, our approach heavily relies on being able to decompose the payoff of the claim into payoffs of shorter maturity. The following broad definition attempts to give a more precise meaning to this concept: 
Definition 5.1. Consider a family of payoffs $\left\{\Sigma_{t, T}\right\}_{0 \leq t \leq T}$, where for each $0 \leq t \leq T, \Sigma_{t, T}$ represents a payoff depending on the path of $X$ on $[t, T]$. We write $\Sigma_{t, T}:=\Sigma\left(\left\{X_{s}\right\}_{t \leq s \leq T}\right)$. We say that the family $\left\{\Sigma_{t, T}\right\}_{0 \leq t \leq T}$ is self-decomposable if, for any $0<t<t^{\prime}<T$, the following decomposition holds true:

$$
\Sigma_{t, T}=f\left(\Sigma_{t, t^{\prime}}\right)+g\left(\Sigma_{t, t^{\prime}}\right) \Sigma_{t^{\prime}, T}
$$

for some measurable functions $f, g: \mathbb{R} \rightarrow \mathbb{R}$.

We now proceed to describe our method. Following our strategy for simple claims, it is natural that a feasible procedure to price a self-decomposable claim $\Sigma_{t, T}(X) \equiv \Sigma\left(\left\{X_{s}\right\}_{t \leq s \leq T}\right)$ will consist of the following two general steps:

(Decomposition) Fix $\delta=(T-t) / k$ for a positive integer $k$ and apply the following decomposition with $t^{\prime}:=t+\delta$ :

$$
\begin{aligned}
& \mathbb{E}^{\widetilde{\mathbb{Q}}}\left[e^{I_{t, T}} \Sigma_{t, T} \mid X_{t}=e_{i}\right]=\mathbb{E}^{\widetilde{\mathbb{Q}}}\left[e^{I_{t, t^{\prime}}} f\left(\Sigma_{t, t^{\prime}}\right) \mathbb{E}^{\widetilde{\mathbb{Q}}}\left[e^{I_{t^{\prime}, T}} \mid \mathcal{F}_{t^{\prime}}\right] \mid X_{t}=e_{i}\right]+\mathbb{E}^{\widetilde{\mathbb{Q}}}\left[e^{I_{t, t^{\prime}}} g\left(\Sigma_{t, t^{\prime}}\right) \mathbb{E}^{\widetilde{\mathbb{Q}}}\left[e^{\left.I_{t^{\prime}, T} \Sigma_{t^{\prime}, T} \mid \mathcal{F}_{t^{\prime}}\right]} \mid X_{t}=e_{i}\right]\right. \\
& =: \mathbb{E}^{\widetilde{\mathbb{Q}}}\left[e^{I_{t, t^{\prime}}} f\left(\Sigma_{t, t^{\prime}}\right) \Xi\left(X_{t^{\prime}}\right) \mid X_{t}=e_{i}\right]+\mathbb{E}^{\widetilde{\mathbb{Q}}}\left[e^{I_{t, t^{\prime}}} g\left(\Sigma_{t, t^{\prime}}\right) \widetilde{\Xi}\left(X_{t^{\prime}}\right) \mid X_{t}=e_{i}\right],
\end{aligned}
$$

where $\Xi\left(e_{l}\right):=\mathbb{E}^{\widetilde{\mathbb{Q}}}\left[e^{I_{t^{\prime}, T}} \mid X_{t^{\prime}}=e_{l}\right]$ and $\widetilde{\Xi}\left(e_{l}\right):=\mathbb{E}^{\widetilde{\mathbb{Q}}}\left[e^{I_{t^{\prime}, T} \Sigma_{t^{\prime}, T}} \mid X_{t^{\prime}}=e_{l}\right]$. We then repeat the above decomposition to evaluate the payoffs $\Xi(\cdot)$ until $T-t^{\prime}$ is small enough.

(Near-expiration approximation) We proceed to apply an efficient approximation to evaluate claims of the form $\mathbb{E}^{\widetilde{\mathbb{Q}}}\left[e^{I_{t, t^{\prime}}} h\left(\Sigma_{t, t^{\prime}}\right) \Xi\left(X_{t^{\prime}}\right) \mid X_{t}=e_{i}\right]$, when $t$ is close to $t^{\prime}$.

Remark 5.7. The above method can also be extended to deal with claims whose payoffs can be decomposed in terms of the payoffs of other types of claims. For instance, we can say that two families of payoffs, say $\left\{\Sigma_{t, T}^{(0)}\right\}_{0 \leq t \leq T}$ and $\left\{\Sigma_{t, T}^{(1)}\right\}_{0 \leq t \leq T}$, are mutually self-decomposable if, for any $t<t^{\prime}<T$,

$$
\Sigma_{t, T}^{(k)}=f_{k}\left(\Sigma_{t, t^{\prime}}^{(0)}\right)+g_{k}\left(\Sigma_{t, t^{\prime}}^{(0)}\right) \Sigma_{t^{\prime}, T}^{(0)}+h_{k}\left(\Sigma_{t, t^{\prime}}^{(1)}\right)+\ell_{k}\left(\Sigma_{t, t^{\prime}}^{(1)}\right) \Sigma_{t^{\prime}, T}^{(1)},
$$

for each $k=0,1$, and some measurable functions $f_{k}, g_{k}, h_{k}, \ell_{k}$.

As an illustration, we now consider the risk-neutral pricing of European barrier and digital contracts written on the volatility process $\left(\sigma_{t}\right)_{t}$. One may view the process $\sigma_{t}:=\sigma^{\prime} X_{s}$ as a proxy to the volatility of a market index. Instruments written on this process may be used to hedge volatility risk associated with periods of macro-economic bust or boom akin to that experienced by the U.S. economy leading into the 2008 crisis. Let us define the following family of path-dependent payoffs:

$$
\Sigma_{t, T}:=\mathbf{1}_{\left\{\max _{t \leq s \leq T} \sigma^{\prime} X_{s} \geq B\right\}}, \quad \text { and } \quad \widetilde{\Sigma}_{t, T}:=\mathbf{1}_{\left\{\max _{t \leq s \leq T} \sigma^{\prime} X_{s}<B\right\}} .
$$

The following simple relationships show that the family of payoffs $\left\{\Sigma_{t, T}\right\}_{0 \leq t \leq T}$ and $\left\{\widetilde{\Sigma}_{t, T}\right\}_{0 \leq t \leq T}$ are self-decomposable:

$$
\text { (1) } \widetilde{\Sigma}_{t, T}=\widetilde{\Sigma}_{t, t^{\prime}} \widetilde{\Sigma}_{t^{\prime}, T}, \quad \text { (2) } \Sigma_{t, T}=\Sigma_{t, t^{\prime}}+\Sigma_{t^{\prime}, T}-\Sigma_{t, t^{\prime}} \Sigma_{t^{\prime}, T}=\Sigma_{t, t^{\prime}}+\left(1-\Sigma_{t, t^{\prime}}\right) \Sigma_{t^{\prime}, T}, \quad\left(t<t^{\prime}<T\right) .
$$

Now, let us consider the following European knock-out style barrier option:

$$
\Psi_{i}^{O U T}[\Xi](t ; T):=\mathbb{E}^{\mathbb{Q}}\left[e^{-\int_{t}^{T} r_{s} d s} \widetilde{\Sigma}_{t, T} \Xi\left(X_{T}\right) \mid X_{t}=e_{i}\right]=\mathbb{E}^{\widetilde{\mathbb{Q}}}\left[e^{\hat{I}_{t, T}} \widetilde{\Sigma}_{t, T} \Xi\left(X_{T}\right) \mid X_{t}=e_{i}\right],
$$

where we had used the same change of probability measure $\widetilde{\mathbb{Q}}$ as in 23 and the following process analog to 44 :

$$
\widehat{I}_{u, v}:=-\int_{u}^{v} r^{\prime} X_{s} d s-\sum_{s \in(u, v]: \Delta X_{s} \neq 0} X_{s^{-}}^{\prime} \tilde{K} X_{s} .
$$


Then, the following decomposition follows from 60 :

$$
\begin{aligned}
& \Psi_{i}^{O U T}[\Xi](t ; T)=\mathbb{E}^{\widetilde{\mathbb{Q}}}\left[e^{\widehat{I}_{t, t^{\prime}}} \widetilde{\Sigma}_{t, t^{\prime}} \mathbb{E}^{\widetilde{\mathbb{Q}}}\left[\widetilde{\Sigma}_{t^{\prime}, T} \Xi\left(X_{T}\right) e^{\widehat{I}_{t^{\prime}, T}} \mid \mathcal{F}_{t^{\prime}}\right] \mid X_{t}=e_{i}\right] \\
& =\mathbb{E}^{\widetilde{\mathbb{Q}}}\left[e^{\widehat{I}_{t, t^{\prime}}} \widetilde{\Sigma}_{t, t^{\prime}} \Psi_{C_{t^{\prime}}}^{O U T}[\Xi]\left(t^{\prime} ; T\right) \mid X_{t}=e_{i}\right]=\Psi_{i}^{O U T}\left[\Psi_{C_{t^{\prime}}}^{O U T}[\Xi]\left(t^{\prime} ; T\right)\right]\left(t ; t^{\prime}\right) .
\end{aligned}
$$

For a near-expiration approximation method for $\Psi_{i}^{O U T}[\Xi]\left(t ; t^{\prime}\right)$ (i.e. when $\zeta:=t^{\prime}-t \approx 0$ ), we use again 25 , 26), and 41):

$$
\Psi_{i}^{O U T}[\Xi]\left(t ; t^{\prime}\right)=e^{-\zeta} \sum_{m=0}^{M-1} \frac{\zeta^{m}}{m !} \Phi_{i, m}^{O U T}(\zeta)
$$

with

$$
\Phi_{i, m}^{O U T}(\zeta)=\frac{1}{(N-1)^{m}} \sum_{\substack{\left(\tilde{e}_{1}, \ldots, \tilde{e}_{m}\right) \\ \tilde{e}_{i} \neq \tilde{e}_{i+1}}} \Xi\left(\tilde{e}_{m}\right) \mathbf{1}_{\left\{\max _{j}\left(\sigma^{\prime} \tilde{e}_{j}\right)<B\right\}} e^{-\zeta \tilde{r}^{\prime} \tilde{e}_{m}-\sum_{n=1}^{m} \tilde{e}_{n-1}^{\prime} \tilde{K}_{\tilde{e}_{n}}} \mathcal{L}_{m}\left(\zeta \tilde{r}^{\prime}\left(\tilde{e}_{0}-\tilde{e}_{m}\right), \ldots, \zeta \tilde{r}^{\prime}\left(\tilde{e}_{m-1}-\tilde{e}_{m}\right)\right)
$$

Note that the knock-in style barrier option $\Psi_{i}^{I N}[\Xi](t ; T):=\mathbb{E}^{\mathbb{Q}}\left[\exp \left\{-\int_{t}^{T} r_{s} d s\right\} \Sigma_{t, T} \Xi\left(X_{T}\right) \mid X_{t}=e_{i}\right]$ can be easily computed using the relation $\widetilde{\Sigma}_{t, T}=1-\Sigma_{t, T}$. In general one only needs to price either a knock-in or a knock-out contract, as the value of the other follows immediately from the knock-in/knock-out parity.

\subsection{Pricing of vulnerable call/put options}

We now develop a new method to price vulnerable call/put options on the stock. The latter type of options have received growing interest in the literature (see the introduction for further references). We set the terminal payoff $\varrho\left(S_{T}\right)=\left(S_{T}-K\right)^{+}$in Eq. (36), thus obtaining a vulnerable time $t$ call option price:

$$
\Pi_{i}(t ; s):=\Pi_{i}(t, T ; s)=\mathbb{E}^{\mathbb{Q}}\left[\left(S_{T}-K\right)_{+} e^{-\int_{t}^{T}\left(r_{s}+h_{s} L_{s}\right) d s} \mid X_{t}=e_{i}, S_{t}=s\right] .
$$

As before, we will change the probability measure into $\widetilde{\mathbb{Q}}$ so that, in terms of the process $I_{t, T}$ defined in 44 ,

$$
\Pi_{i}(t, T ; s)=\mathbb{E}^{\widetilde{\mathbb{Q}}}\left[\left(S_{T}-K\right)_{+} e^{I_{t, T}} \mid X_{t}=e_{i}, S_{t}=s\right] .
$$

Under the risk-neutral measure defined by Eqs. (34) and (35), we have that the stock price is given by

$$
S_{T}=S_{t} \exp \left\{\int_{t}^{T} \tilde{b}^{\prime} X_{s} d s+\int_{t}^{T} \sigma^{\prime} X_{s} d W_{s}^{\mathbb{Q}}\right\}
$$

where the evolution of $X_{t}$ is determined by the risk-neutral generator $A^{\tilde{\mathbb{Q}}}$ under $\tilde{\mathbb{Q}}$ (see, e.g., Elliott et al. (2005)). Above, $\tilde{b}:=\left(\tilde{b}_{1}, \ldots, \tilde{b}_{N}\right)^{\prime}$ is given by $\tilde{b}_{i}:=r_{i}-\sigma_{i}^{2} / 2$. In particular, given $\sigma\left(X_{u}: t \leq u \leq T\right)$ and $S_{t}=s$, we can see $\left\{S_{u}\right\}_{t \leq u \leq T}$ as a geometric Brownian motion with a deterministic time-varying volatility and initial value $s$. As it is well-known, one can express the call price for such a model in terms of the Black-Scholes formula with constant volatility $\bar{\sigma}$, short-rate $r$, spot price $s_{0}$, maturity $\zeta$, and strike $K$ :

$$
\operatorname{BS}\left(\zeta ; s_{0}, \bar{\sigma}^{2}, r, K\right):=e^{-r \zeta} \mathbb{E}^{\widetilde{Q}}\left(s_{0} e^{\bar{\sigma} W_{\zeta}^{\mathbb{Q}}+\left(r-\bar{\sigma}^{2} / 2\right) \zeta}-K\right)_{+} .
$$

Concretely, denoting

$$
\zeta:=T-t, \quad \bar{\sigma}^{2}:=\frac{1}{T-t} \int_{t}^{T}\left(\sigma^{\prime} X_{u}\right)^{2} d u, \quad \bar{b}:=\frac{1}{T-t} \int_{t}^{T} \tilde{b}^{\prime} X_{u} d u, \quad s_{0}:=s e^{\bar{b} \zeta+\zeta \bar{\sigma}^{2} / 2}=s e^{\int_{t}^{T} r^{\prime} X_{u} d u},
$$


we have

$$
\mathbb{E}^{\widetilde{\mathbb{Q}}}\left[\left(S_{T}-K\right)_{+} \mid \sigma\left(X_{u}: t \leq u \leq T\right), S_{t}=s\right]=\mathbb{E}^{\widetilde{\mathbb{Q}}}\left[\left(s e^{\bar{b} \zeta+\zeta \bar{\sigma}^{2} / 2} \times e^{\bar{\sigma} W_{\zeta}^{\mathbb{Q}}-\zeta \bar{\sigma}^{2} / 2}-K\right)_{+}\right]=\operatorname{BS}\left(\zeta ; s_{0}, \bar{\sigma}^{2}, 0, K\right) .
$$

Then, we obtain

$$
\begin{aligned}
\Pi_{i}(t, T ; s) & =\mathbb{E}^{\widetilde{\mathbb{Q}}}\left[\mathbb{E}^{\widetilde{\mathbb{Q}}}\left[e^{I_{t, T}}\left(S_{T}-K\right)_{+} \mid \sigma\left(X_{u}: t \leq u \leq T\right)\right] \mid X_{t}=e_{i}, S_{t}=s\right] \\
& =\mathbb{E}^{\widetilde{\mathbb{Q}}}\left[e^{I_{t, T}} \operatorname{BS}\left(\zeta ; s_{0}, \bar{\sigma}^{2}, 0, K\right) \mid X_{t}=e_{i}, S_{t}=s\right] .
\end{aligned}
$$

Let us now focus on the time-invariant case, where $A^{\tilde{\mathbb{Q}}}$ is time-invariant and, hence, $\Pi_{i}(t, T ; s)=\Pi_{i}(0, T-t ; s)$. We set

$F\left(\zeta ; s, e_{i}\right):=\mathbb{E}^{\mathbb{Q}}\left[\left(S_{\zeta}-K\right)_{+} \mid S_{0}=s, X_{0}=e_{i}\right]=\mathbb{E}^{\widetilde{\mathbb{Q}}}\left[e^{I_{0, \zeta}} \mathrm{BS}\left(\zeta ; s e^{\int_{0}^{\zeta} r^{\prime} X_{u} d u}, \zeta^{-1} \int_{0}^{\zeta}\left(\sigma^{\prime} X_{u}\right)^{2} d u, 0, K\right) \mid S_{0}=s, X_{0}=e_{i}\right]$.

Our approach is based on two ideas. Firstly, if $\zeta$ is small, note that

$$
F\left(\zeta ; s, e_{i}\right) \approx \widetilde{F}\left(\zeta ; s, e_{i}\right):=\mathbb{E}^{\widetilde{\mathbb{Q}}}\left[e^{I_{0, \zeta}} \operatorname{BS}\left(\zeta ; s e^{\zeta r^{\prime} X_{\zeta}},\left(\sigma^{\prime} X_{\zeta}\right)^{2}, 0, K\right) \mid S_{0}=s, X_{0}=e_{i}\right],
$$

up to an error $O(\zeta)$. This is because $\int_{0}^{\zeta} r^{\prime} X_{u} d u=\zeta r^{\prime} X_{\zeta}$ and $\int_{0}^{\zeta}\left(\sigma^{\prime} X_{u}\right)^{2} d u=\zeta\left(\sigma^{\prime} X_{\zeta}\right)^{2}$ if there are no transitions of the process $\left(X_{t}\right)$ during $[0, \zeta]$. Since the expression in 620 can be seen as a European claim of the form 20 with maturity $\zeta$ and payoff $\Xi\left(e_{j}\right):=\mathrm{BS}\left(\zeta ; s e^{\zeta r_{j}}, \sigma_{j}^{2}, 0, K\right)$, one can evaluate this "first order approximation" using our Algorithm 1 in Appendix B.

For a general maturity $\zeta$, we proceed as in 45 . Concretely, for $\delta<\zeta$, we have the recursive relationship

$$
F\left(\zeta ; s, e_{i}\right)=\mathbb{E}^{\widetilde{\mathbb{Q}}}\left[e^{I_{0, \delta}} F\left(\zeta-\delta ; S_{\delta}, X_{\delta}\right) \mid S_{0}=s, X_{0}=e_{i}\right]=\mathbb{E}^{\widetilde{\mathbb{Q}}}\left[e^{I_{0, \delta}} F\left(\zeta-\delta ; s e^{\bar{b} \delta+\bar{\sigma} \sqrt{\delta} W_{1}^{\mathbb{Q}}}, X_{\delta}\right) \mid X_{0}=e_{i}\right],
$$

where now $\bar{\sigma}^{2}:=(\zeta-\delta)^{-1} \int_{\delta}^{\zeta}\left(\sigma^{\prime} X_{u}\right)^{2} d u$ and $\bar{b}:=(\zeta-\delta)^{-1} \int_{\zeta}^{\delta} \tilde{b}^{\prime} X_{u} d u$. As before, the last expression is approximated by

$$
\begin{aligned}
\hat{F}\left(\zeta ; s, e_{i}\right) & :=\mathbb{E}^{\widetilde{\mathbb{Q}}}\left[e^{I_{0, \delta}} F\left(\zeta-\delta ; s e^{\delta \tilde{b}^{\prime} X_{\delta}+\sigma^{\prime} X_{\delta} \sqrt{\delta} W_{1}^{\mathbb{Q}}}, X_{\delta}\right) \mid X_{0}=e_{i}\right] \\
& =\mathbb{E}^{\widetilde{\mathbb{Q}}}\left[e^{I_{0, \delta}} \int_{-\infty}^{\infty} F\left(\zeta-\delta ; s e^{\delta \tilde{b}^{\prime} X_{\delta}+\sqrt{\delta} \sigma^{\prime} X_{\delta} z}, X_{\delta}\right) \frac{1}{\sqrt{2 \pi}} e^{-z^{2} / 2} d z \mid X_{0}=e_{i}\right] .
\end{aligned}
$$

In principle, for a fixed $s$, one can see the right-hand side above as the price of European claim of the form 20 with maturity $\delta$ and payoff $\Xi\left(e_{j}\right):=\mathbb{E}^{\widetilde{\mathbb{Q}}}\left[F\left(\zeta-\delta ; s e^{\delta \tilde{b}_{j}+\sigma_{j} \sqrt{\delta} W_{1}^{\mathrm{Q}}}, e_{j}\right)\right]$. But, since this approach would require to compute $F\left(\zeta-\delta ; p, e_{j}\right)$ for all $p$ and $e_{j}$, this would be computationally inefficient. To resolve this issue, we restrict all possible initial prices $s$ to be in the lattice $\mathcal{L}_{\Delta, B}:=\left\{s e^{i \Delta}: i \in\{-B,-B+1, \ldots, B-1, B\}\right\}$ for a small $\Delta$ and a positive integer $B$. Then, we can approximate $F\left(\zeta ; s e^{i \Delta}, e_{j}\right)$ as follows:

$$
F\left(\zeta ; s e^{i \Delta}, e_{i}\right) \approx \mathbb{E}^{\widetilde{\mathbb{Q}}}\left[e^{I_{0, \delta}} \widetilde{\Xi}_{i}\left(X_{\delta}\right) \mid X_{0}=e_{i}\right],
$$

with

$$
\widetilde{\Xi}_{i}\left(e_{j}\right):=\sum_{k=-B}^{B} F\left(\zeta-\delta ; s e^{k \Delta}, e_{j}\right) \int_{z_{k-1}^{i, j}}^{z_{k}^{i, j}} \frac{1}{\sqrt{2 \pi}} e^{-z^{2} / 2} d z
$$

where

$$
z_{k}^{i, j}:=\frac{(k-i+1 / 2) \Delta-\delta \tilde{b}_{j}}{\sigma_{j} \sqrt{\delta}}, \quad(k=-B,-B+1, \ldots, B-1), \quad z_{-B-1}^{i, j}:=-\infty, \quad z_{B}^{i, j}:=\infty .
$$

Note that the points $z_{k}^{i, j}$ 's are chosen so that the midpoint $\bar{z}_{k}^{i, j}$ of the interval $\left[z_{k-1}^{i, j}, z_{k}^{i, j}\right]$ is such that $s \exp \{k \Delta\}=$ $s \exp \left\{i \Delta+\delta \tilde{b}_{j}+\sqrt{\delta} \sigma_{j} \bar{z}_{k}^{i, j}\right\}$ and, hence, $\widetilde{\Xi}_{i}$ above is a Riemann-Stieltjes sum approximation of the payoff

$$
\Xi_{i}\left(e_{j}\right):=\int_{-\infty}^{\infty} F\left(\zeta-\delta ; s e^{i \Delta+\delta \tilde{b}_{j}+\sqrt{\delta} \sigma_{j} z}, e_{j}\right) \frac{1}{\sqrt{2 \pi}} e^{-z^{2} / 2} d z
$$




\section{Numerical Simulations}

The objective of this section is to assess the accuracy and computational speed of the different methods introduced in the previous section. We first describe the simulation scenario in Section 6.1 and then provide the numerical results in Section 6.2 .

\subsection{Simulation Scenario}

Throughout this part, we use the following parameter specifications borrowed from the parameter setup of Capponi and Figueroa-López (2012). The right panel also shows the loss rates corresponding to three distinct regimes, hereafter referred to as "low", "middle", and "high" default regime. The default intensities are based on the empirical result of Giesecke et al. (2011) . In agreement with empirical market evidence, we have selected the loss rates to be increasing in the credit riskiness of the regime.

\begin{tabular}{|c|c|c|c|}
\hline$a_{i, j}^{\mathbb{Q}}$ & 1 (low) & 2 (middle) & 3 (high) \\
\hline \hline 1 & -0.380313 & 0.33687 & 0.043443 \\
\hline 2 & 0.254397 & -0.254397 & 0 \\
\hline 3 & 0.208683 & 0.000006 & -0.208689 \\
\hline
\end{tabular}

\begin{tabular}{|c|c|c|}
\hline & $\mathrm{h}$ & $\mathrm{L}$ \\
\hline \hline 1 & $0.741 \%$ & $10 \%$ \\
\hline 2 & $4.261 \%$ & $40 \%$ \\
\hline 3 & $11.137 \%$ & $90 \%$ \\
\hline
\end{tabular}

Table 1: Left panel shows the generator of the Markov chain under the risk-neutral measure. The rows indicate the starting state, while the columns indicate the ending state of the chain. The right panel shows the default intensities as reported in Giesecke et al. (2011) as well as our loss rates given default associated to three regimes.

\subsection{Numerical Results}

We start by illustrating the accuracy and computational speed of the method described in Section 5.1 for computing the pre-default price of a defaultable bond. These bond prices, denoted hereafter $\psi(t):=\left(\psi_{1}(t), \ldots, \psi_{N}(t)\right)^{\prime}$, are given by the formula (20) with payoff function $\Xi\left(e_{i}\right) \equiv 1$. Concretely, we define

$$
\psi_{i}(t):=\mathbb{E}^{\mathbb{Q}}\left[e^{-\int_{t}^{T}\left(r_{s}+h_{s} L_{s}\right) d s} \mid X_{t}=e_{i}\right] .
$$

We compute (63) by our method and a standard numerical solution of the Feynman-Kač representation (29) for the bond price (63). Table 2 shows the time-0 bond prices for different maturities computed using our method and a Runge-Kutta type numerical solution ${ }^{3}$ of the system $(29)$ with terminal condition $\Psi_{i}(T)=1$ under the parameter setup of Table 1. Figure 1 shows the time $t$ bond price for maturity $T=1$ year (left panel), and $T=20$ years (right panel). It is evident that our method is highly accurate even for long maturity bonds. Furthermore, according to our computational experiments, our method is in most cases more efficient than either solving the ODE system by the Runge-Kutta algorithm or computing the exponential (33) using a Padé type approximation. For the sake of completeness, Appendix C compares the processor time for these three methods.

Next, we illustrate the power of the algorithm to compute prices of the knock-out digital and call options described at the end of Section 5.3. Table 3 reports the prices of knock-out digital and call options written on the volatility process obtained by applying our methodology, and compares them with the corresponding Monte-Carlo estimates. The results show that our method is highly accurate even for long maturity options. We conclude the numerical analysis with an illustration of the algorithm performance on pricing vulnerable European type claims, which depend

\footnotetext{
${ }^{2}$ Giesecke et al. (2011) employed a three-state homogenous regime-switching model to examine the effects of an array of financial and macro-economic variables in explaining variations in the realized default rates of the U.S. corporate bond market over the course of 150 years.

${ }^{3}$ This solution was obtained using the MATLAB function ode45.
} 


\begin{tabular}{|c|cc|cc|cc|}
\hline & \multicolumn{2}{|c|}{$X_{0}=e_{1}$} & \multicolumn{2}{c|}{$X_{0}=e_{2}$} & \multicolumn{2}{c|}{$X_{0}=e_{3}$} \\
\hline$T$ (yrs.) & NM & ODE & NM & ODE & NM & ODE \\
\hline 0.25 & 0.9921 & 0.9921 & 0.9884 & 0.9884 & 0.9686 & 0.9686 \\
0.50 & 0.9837 & 0.9837 & 0.9772 & 0.9772 & 0.9393 & 0.9393 \\
1.00 & 0.9659 & 0.9659 & 0.9555 & 0.9555 & 0.8864 & 0.8864 \\
2.00 & 0.9282 & 0.9281 & 0.9146 & 0.9146 & 0.7991 & 0.7990 \\
5.00 & 0.8140 & 0.8136 & 0.8029 & 0.8031 & 0.6274 & 0.6273 \\
10.0 & 0.6488 & 0.6484 & 0.6430 & 0.6431 & 0.4701 & 0.4701 \\
15.0 & 0.5170 & 0.5166 & 0.5131 & 0.5131 & 0.3691 & 0.3690 \\
20.0 & 0.4119 & 0.4116 & 0.4090 & 0.4090 & 0.2931 & 0.2930 \\
25.0 & 0.3282 & 0.3280 & 0.3259 & 0.3259 & 0.2334 & 0.2333 \\
30.0 & 0.2616 & 0.2613 & 0.2597 & 0.2597 & 0.1859 & 0.1858 \\
50.0 & 0.1055 & 0.1053 & 0.1047 & 0.1047 & 0.0750 & 0.0749 \\
\hline
\end{tabular}

Table 2: Time $t=0$ bond prices for different time-to-maturities $T$ using the ODE method and the new method (NM) with parameters $M=2$ and $\delta=2.5$ years (see Algorithm 1 in Appendix B).

on both the diffusion and the Markov chain component. Figure 2 compares our novel method with a plain Monte Carlo method, given that the initial regime is 1 (left panel), and 3 (right panel) (regime 2 is quite similar to regime $3)$. As seen there, the new method significantly improves the quality of the approximation compared to the first order approximation (62), especially for longer maturities. For the sake of completeness, we have included the precise algorithm in Appendix B (see Algorithm 2 therein). Error analysis and further extensions of this method will be postponed for a future publication.

\section{Conclusions}

We have rigorously analyzed pricing of vulnerable claims in regime-switching markets, and provided novel semimartingale representations. The main theoretical tool underlying our results is a novel Poisson series representation of a generic claim price, whose payoff depends on the underlying Markov chain. Using this representation, we were able to prove differentiability of the pre-default price function under mild conditions on the Markov chain generator. This allowed us to provide rigorous Feynman-Kač and semimartingale representations of the price process of the vulnerable claim. We then constructed a novel pricing methodology which makes use of the Poisson series representation, and expands the price of the vulnerable claim in terms of the Laplace transform of the symmetric Dirichlet distribution. We provided a detailed error analysis of our proposed algorithm under different sets of assumptions relating to how each conditional price component may be approximated. One of the novel features of our method and error analysis is that it also applies to time-dependent Markov-chain generators. We further demonstrated the robustness of our methodology by showing that it can be used to price path dependent claims, termed self-decomposable, as well as vulnerable European-type options, whose payoff depends on both the diffusion and the Markov-chain component. We numerically demonstrated the algorithm on defaultable bonds, barrier options on the stock volatility, as well as vulnerable European call option prices. In all these cases, we have shown that it achieves a high level of accuracy, and, at the same time, it is computationally fast when compared to standard PDE or Monte-Carlo based methods.

\section{Acknowledgments}

The second author's research was partially supported by a grant from the US National Science Foundation (DMS0906919). The third author's research was partially supported by a Purdue Research Foundation Ph.D. student grant. 

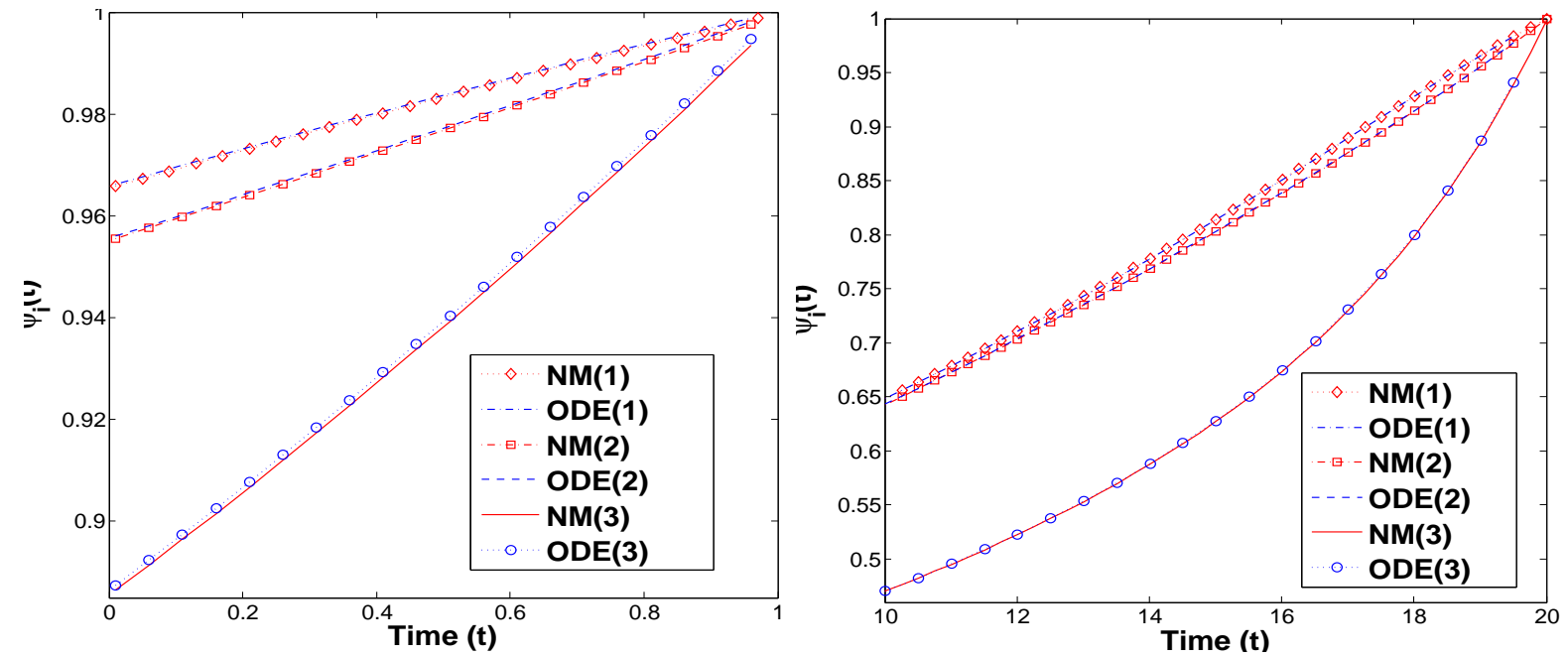

Figure 1: Bond price comparison for a one-year and a 20-year bond using the ODE method and the new method (NM) parameters $M=2$ and $\delta=2.5$ years (see Algorithm 1 in Appendix B).

\section{Vulnerable Call Option Prices given $X_{0}=1$}

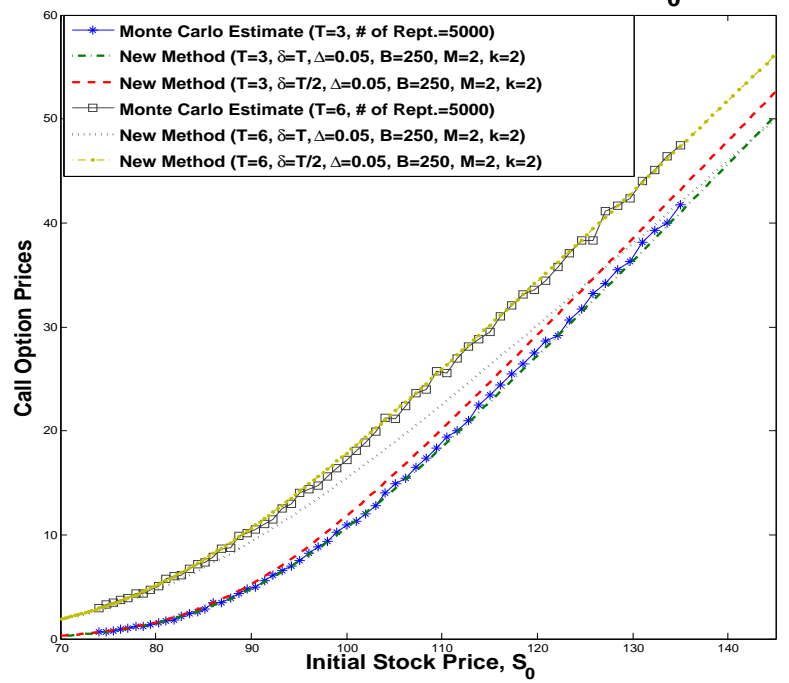

Vulnerable Call Option Prices given $X_{0}=3$

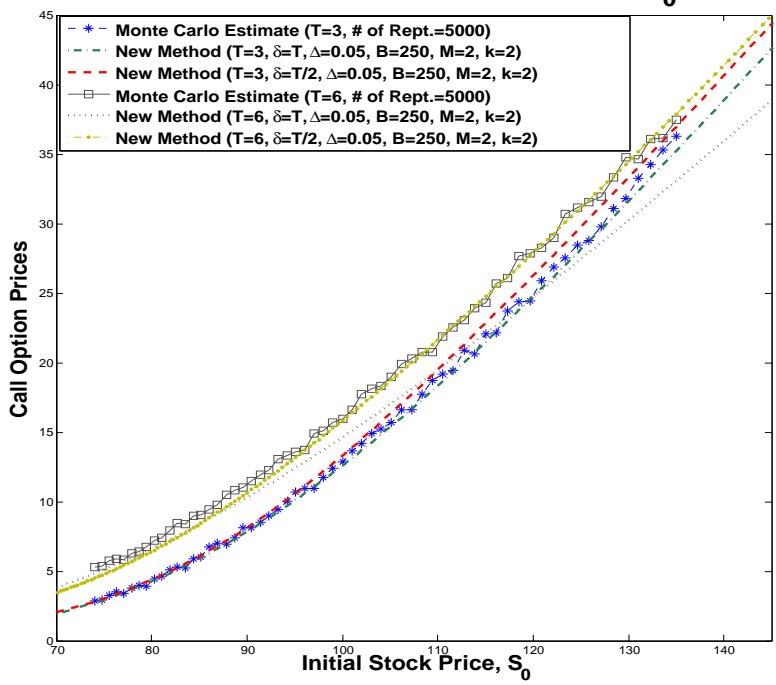

Figure 2: Comparison of vulnerable call option prices using Monte Carlo and the new method of the Algorithm 2 in Appendix B for maturities of $T=3$ years and $T=6$ years. Above, $k=2$ refers to the number of discretization points on the PriceClaim Method of Appendix B(i.e. $\delta=T / k$ therein). 


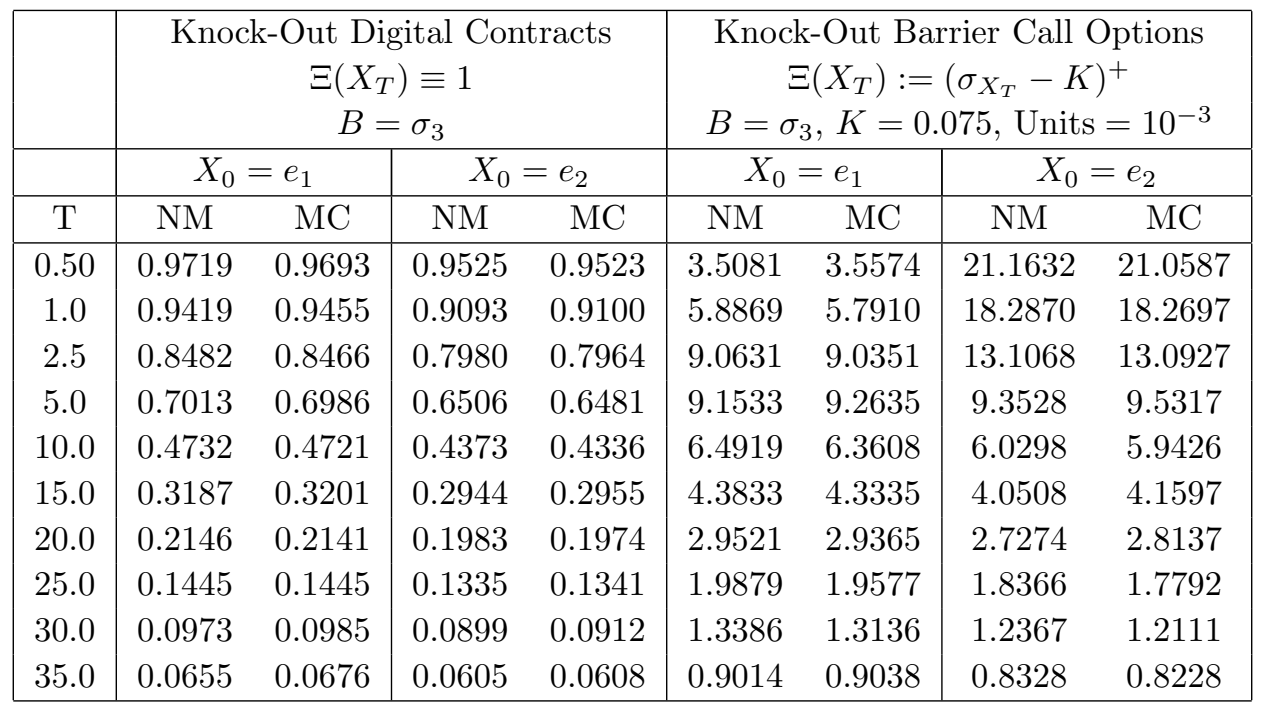

Table 3: Knock-out digital and call options on the volatility process using the new method (NM) and the Monte Carlo method (MC). The call option prices are expressed on the $10^{-3}$ scale and the results for $X_{0}=e_{3}$ have been omitted since they are knocked out at contract initiation. Here, $r=(0.01,0.1,0.3)^{\prime}$ and $\sigma=(0.05,0.1,0.2)^{\prime}$.

The authors gratefully acknowledge the constructive and insightful comments provided by two anonymous referees, which significantly contributed to improve the quality of this manuscript.

\section{A Proofs}

\section{Proof of Lemma 2.2 ,}

By the definition of $\mathbb{Q}$ and the fact that $\eta$ is a $\mathbb{G}$-martingale (see the paragraph before $(15)$ ), it suffices to prove that $\eta_{t} \xi_{t}^{\mathbb{Q}}$ is a $\mathbb{G}$-martingale under $\mathbb{P}$. From Itô's formula and the definition of $\eta$ in 15 , we have the process

$$
\eta_{t} \xi_{t}^{\mathbb{Q}}=\xi_{0}^{\mathbb{Q}}+\int_{0}^{t} \eta_{s^{-}} d \xi_{s}^{\mathbb{Q}}+\int_{0}^{t} \xi_{s^{-}}^{\mathbb{Q}} d \eta_{s}+\sum_{s \leq t} \Delta \xi_{s}^{\mathbb{Q}} \Delta \eta_{s}
$$

From 22,15 , and $19,\left(\eta_{t} \xi_{t}^{\mathbb{Q}}\right)_{t}$ can be written as

$$
\xi_{0}^{\mathbb{Q}}+\int_{0}^{t} \eta_{s^{-}} d \xi_{s}^{\mathbb{Q}}+\int_{0}^{t} \xi_{s^{-}}^{\mathbb{Q}} d \eta_{s}+\sum_{0<s \leq t} \sum_{k, l=1}^{N} \eta_{s^{-}} \kappa_{k, l}(u) \Delta H(s) \Delta H_{s}^{k, l},
$$

where $H_{t}^{k, l}:=\sum_{0<s \leq t} \mathbf{1}_{\left\{X_{s^{-}}=k\right\}} \mathbf{1}_{\left\{X_{s}=l\right\}}$. Since the first two terms on the right-hand side of the previous equality are (local) martingales under $\mathbb{P}$, it remains to show that the last term vanishes. But, given that $\Delta H_{s} \neq 0$ at $s=\tau$, the summation in the last term above will be 0 provided that $\Delta X_{\tau}=0$, a.s. In order to show this, let us recall that by definition $X$ has no fixed-jump times; i.e. $\mathbb{P}\left(\Delta X_{t} \neq 0\right)=0$ for any fixed time $t>0$. Also, using the definition of $\tau$ given in Eq. 11), $\tau=\inf \left\{t \geq 0: \int_{0}^{t} h\left(X_{s}\right) d s \geq \Theta\right\}$, where $\Theta$ is an exponential random variable independent of $X$. Then, conditioning on $X, \mathbb{P}\left(\Delta X_{\tau} \neq 0\right)=\mathbb{E}\left\{\mathbb{E}\left[\mathbf{1}_{\left\{\Delta X_{\tau} \neq 0\right\}} \mid X_{s}, s \geq 0\right]\right\}$. Denoting $0<\tau_{1}<\tau_{2}<\ldots$ the transition times of the Markov chain $X, \mathbb{E}\left[\mathbf{1}_{\left\{\Delta X_{\tau} \neq 0\right\}} \mid X_{s}, s \geq 0\right]$ is given by

$$
\mathbb{E}\left[\sum_{i=1}^{\infty} \mathbf{1}_{\left\{\tau=\tau_{i}\right\}} \mid X_{s}, s \geq 0\right]=\mathbb{E}\left[\sum_{i=1}^{\infty} \mathbf{1}_{\left\{\int_{0}^{\tau_{i}} h\left(X_{s}\right) d s=\Theta\right\}} \mid X_{s}, s \geq 0\right]=0,
$$


where the last equality follows from the independence of $X$ and $\Theta$, and the fact that $\Theta$ is a continuous random variable.

\section{Proof of Theorem 3.1.}

For simplicity we omit the dependence on the claim's payoff $\Xi$ and maturity $T$ in $(20)$ and just write $\Psi_{i}(t)$. Recall that $\Psi(t):=\left(\Psi_{1}(t), \ldots, \Psi_{N}(t)\right)$ is such that

$$
\gamma(t ; T)=\mathbb{E}^{\mathbb{Q}}\left[\Xi\left(X_{T}\right) e^{-\int_{t}^{T}\left(r_{s}+h_{s} L_{s}\right) d s} \mid X_{t}\right]=\left\langle\Psi(t), X_{t}\right\rangle .
$$

But also, changing into the probability measure $\widetilde{\mathbb{Q}}$, we can write

$$
\gamma(t ; T)=\mathbb{E}^{\mathbb{Q}}\left[\Xi\left(X_{T}\right) e^{-\int_{t}^{T}\left(r_{s}+h_{s} L_{s}\right) d s} \mid \mathcal{F}_{t}\right]=\mathbb{E}^{\widetilde{\mathbb{Q}}}\left[\Xi\left(X_{T}\right) e^{-\int_{t}^{T}\left(r_{s}+h_{s} L_{s}\right) d s} \frac{\tilde{\eta}_{t}}{\tilde{\eta}_{T}} \mid \mathcal{F}_{t}\right],
$$

and, hence, we have the following representation for $\Psi_{i}$ :

$$
\Psi_{i}(t)=\mathbb{E}^{\widetilde{\mathbb{Q}}}\left[e^{-\int_{t}^{T}\left(r_{s}+h_{s} L_{s}\right) d s} \frac{\tilde{\eta}_{t}}{\tilde{\eta}_{T}} \mid X_{t}=e_{i}\right] .
$$

The solution of 21) can be written as

$$
\tilde{\eta}_{t}:=e^{-\int_{0}^{t} \sum_{i, j} a_{i, j}^{\mathrm{Q}}(s) \tilde{\kappa}_{i, j}(s) H_{s}^{i} d s+\sum_{0<s \leq t} \log \left(1+\sum_{i, j} \tilde{\kappa}_{i, j}(s) \Delta H_{s}^{i, j}\right)} ;
$$

see pp. 334-335 in Bielecki et al. (2008-b). Let $\widetilde{K}(t)=\left[\widetilde{K}_{i, j}(t)\right]_{i, j}$ and $\tilde{r}(t):=\left(\tilde{r}_{1}(t), \ldots, \tilde{r}_{N}(t)\right)^{\prime}$ be defined by

$$
\widetilde{K}_{i, j}(t):=\log \left(1+\tilde{\kappa}_{i, j}(t)\right) \mathbf{1}_{i \neq j}=-\log \left((N-1) a_{i, j}^{\mathbb{Q}}(t)\right) \mathbf{1}_{i \neq j}, \quad \tilde{r}_{i}(t):=r_{i}+h_{i} L_{i}-\sum_{j=1}^{N} a_{i, j}^{\mathbb{Q}}(t) \tilde{\kappa}_{i, j}(t) .
$$

Then, since by the definition (14), $\Delta H_{s}^{i, j}=1$ whenever there is a jump from the state $e_{i}$ to the state $e_{j}$ (i.e. $X_{s^{-}}=e_{i}$ and $X_{s}=e_{j}$ ) and $\Delta H_{s}^{i, j}=0$, otherwise, we have

$$
\sum_{s \leq t} \log \left(1+\sum_{i, j} \tilde{\kappa}_{i, j}(s) \Delta H_{s}^{i, j}\right)=\sum_{s \leq t} \sum_{i, j} \log \left(1+\tilde{\kappa}_{i, j}(s)\right) \Delta H_{s}^{i, j}=\sum_{s \in(0, t]: \Delta X_{s} \neq 0} X_{s^{-}}^{\prime} \widetilde{K}(s) X_{s} .
$$

Similarly, by the definition (14),

$$
\int_{0}^{t} \sum_{i, j} a_{i, j}^{\mathbb{Q}}(s) \tilde{\kappa}_{i, j}(s) H_{s}^{i} d s=\int_{0}^{t} v(s) X_{s} d s
$$

where $v=\left(v_{1}, \ldots, v_{N}\right)^{\prime}$ with $v_{i}(s):=\sum_{j=1}^{N} a_{i, j}^{\mathbb{Q}}(s) \tilde{\kappa}_{i, j}(s)$. Then, since $r_{s}+h_{s} L_{s}=\hat{r}^{\prime} X_{s}$ with $\hat{r}=\left(r_{1}+h_{1} L_{1}, \ldots, r_{N}+\right.$ $\left.h_{N} L_{N}\right)$, we obtain from 65 that

$$
\Psi_{i}(t)=\mathbb{E}^{\widetilde{\mathbb{Q}}}\left[\exp \left\{-\int_{t}^{T} \tilde{r}(s)^{\prime} X_{s} d s-\sum_{s \in(t, T]: \Delta X_{s} \neq 0} X_{s^{-}}^{\prime} \widetilde{K}(s) X_{s}\right\} \mid X_{t}=e_{i}\right]
$$

where $X^{\prime}$ denotes the transpose of $X$.

\section{Proof of Lemma 4.1.}

Again, for simplicity we omit the dependence on the claim's payoff $\Xi$ and maturity $T$ in the functions $\Psi_{i}[\Xi](t ; T)$ and $\Phi_{i, m}[\Xi](t ; T)$. In light of the series representation $[25)$, we see that it suffices to show the function $\Phi_{i, m}(\zeta)$ is continuously differentiable in $\zeta \in(0, T)$ for each $m \geq 0$, and that there exists a sequence $\left\{K_{m}\right\}_{m \geq 0}$ of reals such that

$$
\text { (i) } \sup _{0 \leq \zeta \leq T}\left|\Phi_{m}(\zeta)\right| \leq K_{m}, \quad \text { (ii) } \sup _{0 \leq \zeta \leq T}\left|\Phi_{m}^{\prime}(\zeta)\right| \leq K_{m}, \quad \text { (iii) } \sum_{m=0}^{\infty} e^{-\zeta} \frac{\zeta^{m}}{m !} K_{m}<\infty .
$$


We now show that (i)-(iii) are satisfied provided the function $\widetilde{K}$ and $\tilde{r}$ defined in 24 are such that

$$
\sup _{s \in[0, T]}\left|\tilde{r}_{i}(s)\right|<\infty, \quad \sup _{s \in[0, T]}\left|\widetilde{K}_{i, j}(s)\right|<\infty, \quad \text { and } \quad \sup _{s \in[0, T]}\left|\widetilde{K}_{i, j}^{\prime}(s)\right|<\infty .
$$

The conditions 66 directly follow from 22$]$ and 28 . Denoting $\mathcal{P}_{m, i}(\zeta)$ the random function inside the expectation $\mathbb{E}_{i}^{\widetilde{Q}}$ in 26 , one can check that

$$
\sup _{0 \leq \zeta \leq T}\left|\mathcal{P}_{m, i}(\zeta)\right| \leq \Xi\left(e_{i}\right) \cdot e^{m T \max _{i} \sup _{0 \leq \zeta \leq T}\left|\tilde{r}_{i}(\zeta)^{\prime}\right|+m \max _{i, j} \sup _{0 \leq \zeta \leq T}\left|\widetilde{K}_{i, j}(\zeta)\right|}=\Xi\left(e_{i}\right) A^{m},
$$

for a constant $A<\infty$. Also, $\mathcal{P}_{m, i}(\zeta)$ is continuously differentiable and

$$
\mathcal{P}_{m, i}^{\prime}(\zeta)=\mathcal{P}_{m, i}(\zeta)\left\{\sum_{n=0}^{m}\left[\bar{U}_{(n+1)} \tilde{r}\left(T-\zeta \bar{U}_{(n+1)}\right)^{\prime} \widetilde{X}_{n}-\bar{U}_{(n)} \tilde{r}\left(T-\zeta \bar{U}_{(n)}\right)^{\prime} \widetilde{X}_{n}\right]-\sum_{n=1}^{m} \widetilde{X}_{n-1}^{\prime} \widetilde{K}^{\prime}\left(T-\zeta \bar{U}_{(n)}\right) \widetilde{X}_{n} \bar{U}_{(n)}\right\},
$$

where $\bar{U}_{(n)}:=1-U_{(n)}$. In particular, there exists a constant $B<\infty$ such that

$$
\sup _{0 \leq \zeta \leq T}\left|\mathcal{P}_{m}^{\prime}(\zeta)\right| \leq B m A^{m} .
$$

By the formal definition of the derivative $\Phi_{m, i}^{\prime}(\zeta)$ and the Dominated Convergence Theorem, one can check that 67)-68 will suffice for (i)-(iii).

\section{Proof of Theorem 4.2 .}

The following three parts show each of the statements of Theorem 4.2 .

(1) Consider the $(\mathbb{F}, \mathbb{Q})$-local martingale

$$
Z(t):=\mathbb{E}^{\mathbb{Q}}\left[\Xi\left(X_{T}\right) e^{-\int_{0}^{T}\left(r_{s}+h_{s} L_{s}\right) d s} \mid \mathcal{F}_{t}\right] .
$$

Note that

$$
Z(t)=e^{-\int_{0}^{t}\left(r_{s}+h_{s} L_{s}\right) d s} \gamma(t)=e^{-\int_{0}^{t}\left(r_{s}+h_{s} L_{s}\right) d s}\left\langle\Psi(t), X_{t}\right\rangle,
$$

and, thus, by Itô's formula and the decomposition (16), we get

$$
\begin{aligned}
d Z(t)= & -\left(r_{t}+h_{t} L_{t}\right) e^{-\int_{0}^{t}\left(r_{u}+h_{u} L_{u}\right) d u}\left\langle\Psi(t), X_{t}\right\rangle d t+e^{-\int_{0}^{t}\left(r_{u}+h_{u} L_{u}\right) d u}\left(\left\langle\frac{d \Psi(t)}{d t}, X_{t}\right\rangle+\left\langle\Psi(t),\left(A^{\mathbb{Q}}\right)_{t}^{\prime} X_{t}\right\rangle\right) d t \\
& +e^{-\int_{0}^{t}\left(r_{u}+h_{u} L_{u}\right) d u}\left\langle\Psi(t), d M^{\mathbb{Q}}(t)\right\rangle,
\end{aligned}
$$

where we have used the fact that $\Psi(t)$ is differentiable in $t$ by virtue of Lemma 4.1. Since $Z(t)$ is a $(\mathbb{F}, \mathbb{Q})$-(local) martingale, its drift term must be zero. When $X_{t}=e_{i}$, such condition translates into the coupled system of differential equations for the price function $\Psi_{i}$ given in Eq. 29,

(2) The following dynamics for $Z$ also follows from 69 and the martingale property of $\left\{Z_{t}\right\}_{t \geq 0}$ :

$$
d Z(t)=e^{-\int_{0}^{t}\left(r_{u}+h_{u} L_{u}\right) d u}\left\langle\Psi(t), d M^{\mathbb{Q}}(t)\right\rangle .
$$

In particular, the price process $\gamma(t)$ follows the dynamics

$$
\begin{aligned}
d \gamma(t) & =d\left(e^{\int_{0}^{t}\left(r_{s}+h_{s} L_{s}\right) d s} Z(t)\right) \\
& =\left(r_{t}+h_{t} L_{t}\right) e^{\int_{0}^{t}\left(r_{s}+h_{s} L_{s}\right) d s} Z(t) d t+e^{\int_{0}^{t}\left(r_{s}+h_{s} L_{s}\right) d s} d Z(t) \\
& =\left(r_{t}+h_{t} L_{t}\right) e^{\int_{0}^{t}\left(r_{s}+h_{s} L_{s}\right) d s} Z(t) d t+\left\langle\Psi(t), d M^{\mathbb{Q}}(t)\right\rangle .
\end{aligned}
$$


Next, denoting $\tilde{H}(t):=1-H(t)=\mathbf{1}_{\tau>t}$ and applying Itô's formula,

$$
d \Gamma(t ; T)=d(\tilde{H}(t) \gamma(t))=\tilde{H}\left(t^{-}\right) d \gamma(t)+\gamma\left(t^{-}\right) d \tilde{H}(t)+\Delta \gamma(t) \Delta \tilde{H}(t) .
$$

By the continuity of $\Psi(t)$,

$$
\Delta \gamma(t) \Delta \tilde{H}_{t}=\left\langle\Psi(t), \Delta X_{t}\right\rangle \Delta \tilde{H}_{t}=\left\langle\Psi(t), \Delta X_{\tau}\right\rangle=0, \quad \text { a.s. },
$$

where the last equality hold true in light of the construction (1) and the independence of $X$ and the exponential variable $\Theta$ there (see also the proof of Lemma 2.2 above). Combining (70) and (71),

$$
\begin{aligned}
d \Gamma(t ; T) & =\tilde{H}\left(t^{-}\right)\left(r_{t}+h_{t} L_{t}\right) e^{\int_{0}^{t}\left(r_{s}+h_{s} L_{s}\right) d s} Z(t) d t+\tilde{H}\left(t^{-}\right)\left\langle\Psi(t), d M^{\mathbb{Q}}(t)\right\rangle+\gamma\left(t^{-}\right) d \tilde{H}(t) \\
& =\left(r_{t}+h_{t} L_{t}\right) \Gamma(t ; T) d t+\tilde{H}\left(t^{-}\right)\left\langle\Psi(t), d M^{\mathbb{Q}}(t)\right\rangle-\gamma\left(t^{-}\right) d H(t)
\end{aligned}
$$

By the Doob-Meyer decomposition of Lemma 2.2 we have $d H(t)=d \xi_{t}^{\mathbb{Q}}+\left(1-H\left(t^{-}\right)\right) h_{t} d t$ and, thus,

$$
d \Gamma(t ; T)=\left(\left[r_{t}+h_{t} L_{t}\right] \Gamma(t ; T)-\gamma\left(t^{-}\right) h_{t}\left[1-H\left(t^{-}\right)\right]\right) d t+\tilde{H}\left(t^{-}\right)\left\langle\Psi(t), d M^{\mathbb{Q}}(t)\right\rangle-\gamma\left(t^{-}\right) d \xi_{t}^{\mathbb{Q}}
$$

The last step follows from the fact that on the event $\tau>t$, we have $\Gamma\left(t^{-} ; T\right)=\gamma\left(t^{-} ; T\right)$, by definition of $\Gamma(t, T)$, and also $\gamma\left(t^{-}\right)=\left\langle\Psi(t), X_{t^{-}}\right\rangle$. Therefore, we can write the risk-neutral dynamics of prices process $\Gamma(t ; T)$ as

$$
\frac{d \Gamma(t ; T)}{\Gamma\left(t^{-} ; T\right)}=\left(r_{t}+h_{t}\left(L_{t}-1\right)\right) d t+\frac{\left\langle\Psi(t), d M^{\mathbb{Q}}(t)\right\rangle}{\left\langle\Psi(t), X_{t^{-}}\right\rangle}-d \xi_{t}^{\mathbb{Q}}, \quad t<\tau
$$

which concludes the second statement of the theorem.

(3) The price dynamics of the vulnerable claim under the real-world measure follows directly from plugging Eq. (17) into 72 to get:

$$
\frac{d \Gamma(t, T)}{\Gamma\left(t^{-}, T\right)}=\left(r_{t}+h_{t}\left(L_{t}-1\right)+\frac{\left\langle\Psi(t),\left(A^{\prime}(t)-\left(A^{\mathbb{Q}}\right)^{\prime}(t)\right) X_{t}\right\rangle}{\left\langle\Psi(t), X_{t}\right\rangle}\right) d t+\frac{\left\langle\Psi(t), d M^{\mathbb{P}}(t)\right\rangle}{\left\langle\Psi(t), X_{t^{-}}\right\rangle}-d \xi_{t}^{\mathbb{P}},
$$

where we also used that $\xi^{\mathbb{P}}=\xi^{\mathbb{Q}}$ (see Lemma 2.2. Finally, we get the dynamics 31 since

$$
\frac{\left\langle\Psi(t),\left(A^{\prime}(t)-\left(A^{\mathbb{Q}}\right)^{\prime}(t)\right) X_{t}\right\rangle}{\left\langle\Psi(t), X_{t}\right\rangle}=\frac{\left\langle\left(A(t)-A^{\mathbb{Q}}(t)\right) \Psi(t), X_{t}\right\rangle}{\left\langle\Psi(t), X_{t}\right\rangle} .
$$

When $X_{t}=e_{i}$, the previous quantity simplifies as

$$
D_{i}(t):=\sum_{j=1}^{n}\left(a_{i, j}(t)-a_{i, j}^{\mathbb{Q}}(t)\right) \frac{\Psi_{j}(t)}{\Psi_{i}(t)}
$$

and 73 can be written as $\left\langle\left(D_{1}(t), \ldots, D_{N}(t)\right)^{\prime}, X_{t}\right\rangle$.

\section{Proof of Theorem 4.4 .}

The following three parts show each of the statements of Theorem 4.4

(1) Consider the $(\mathbb{F}, \mathbb{Q})$-local martingale

$$
Z\left(t, S_{t}\right):=\mathbb{E}^{\mathbb{Q}}\left[\varrho\left(S_{T}\right) e^{-\int_{0}^{T}\left(r_{s}+h_{s} L_{s}\right) d s} \mid \mathcal{F}_{t}\right] .
$$

Note that

$$
Z\left(t, S_{t}\right)=e^{-\int_{0}^{t}\left(r_{s}+h_{s} L_{s}\right) d s} \gamma\left(t ; S_{t}\right)=e^{-\int_{0}^{t}\left(r_{s}+h_{s} L_{s}\right) d s}\left\langle\Pi\left(t ; S_{t}\right), X_{t}\right\rangle,
$$


where we used the notation $\gamma\left(t ; S_{t}\right)=\mathbb{E}^{\mathbb{Q}}\left[\varrho\left(S_{T}\right) e^{-\int_{t}^{T}\left(r_{s}+h_{s} L_{s}\right) d s} \mid \mathcal{F}_{t}\right]$. Thus, by Itô's formula, we get

$$
d Z\left(t, S_{t}\right)=-\left(r_{t}+h_{t} L_{t}\right) e^{-\int_{0}^{t}\left(r_{u}+h_{u} L_{u}\right) d u}\left\langle\Pi\left(t ; S_{t}\right), X_{t}\right\rangle d t+e^{-\int_{0}^{t}\left(r_{u}+h_{u} L_{u}\right) d u} d\left\langle\Pi\left(t ; S_{t}\right), X_{t}\right\rangle .
$$

Under the stated differentiability assumptions, we have that

$$
d\left\langle\Pi\left(t ; S_{t}\right), X_{t}\right\rangle=\mathcal{L}\left\langle\Pi\left(t ; S_{t}\right), X_{t}\right\rangle d t+d \mathcal{M}_{t},
$$

where $\mathcal{L}$ is the generator of $\left(t, S_{t}, C_{t}\right)$, and $\left\{\mathcal{M}_{t}\right\}_{t \geq 0}$ is the local martingale component of $\left(t, S_{t}, C_{t}\right)$, under the risk neutral measure $\mathbb{Q}$. These have been given in a more general context in Capponi and Figueroa-López (2012), Appendix A. In our specific setting, they reduce to

$$
\mathcal{L} \Pi_{i}(t ; s)=\frac{\partial \Pi_{i}(t ; s)}{\partial t}+r_{i} s \frac{\partial \Pi_{i}(t ; s)}{\partial s}+\frac{\sigma_{i}^{2} s^{2}}{2} \frac{\partial^{2} \Pi_{i}(t ; s)}{\partial^{2} s}+\sum_{j \neq i} a_{i, j}^{\mathbb{Q}}(t)\left(\Pi_{j}(t ; s)-\Pi_{i}(t ; s)\right),
$$

and

$$
d \mathcal{M}_{t}=\sum_{i=1}^{N}\left\{\sum_{j \neq i}\left[\Pi_{j}\left(t ; S_{t-}\right)-\Pi_{i}\left(t ; S_{t-}\right)\right] d M_{t}^{i, j}+\sigma_{i} S_{t} \frac{\partial \Pi_{i}\left(t ; S_{t-}\right)}{\partial s} H_{t}^{i} d W_{t}^{\mathbb{Q}}\right\} .
$$

where $H_{t}^{i}$ and $M_{t}^{i, j}$ have been defined in Eqs. (14) and (13), respectively. Using the operator $\mathcal{L}$ and local martingale $\mathcal{M}_{t}$ given by Eq. 75 and (76), respectively, we obtain

$$
\begin{aligned}
d Z\left(t, S_{t}\right)= & -\left(r_{t}+h_{t} L_{t}\right) e^{-\int_{0}^{t}\left(r_{u}+h_{u} L_{u}\right) d u}\left\langle\Pi\left(t ; S_{t}\right), X_{t}\right\rangle d t+e^{-\int_{0}^{t}\left(r_{u}+h_{u} L_{u}\right) d u}\left[\left(\mathcal{L}\left\langle\Pi\left(t ; S_{t}\right), X_{t}\right\rangle\right) d t+d \mathcal{M}_{t}\right] \\
= & e^{-\int_{0}^{t}\left(r_{u}+h_{u} L_{u}\right) d u}\left[-\left(r_{t}+h_{t} L_{t}\right)\left\langle\Pi\left(t ; S_{t}\right), X_{t}\right\rangle+\frac{\partial\left\langle\Pi\left(t ; S_{t}\right), X_{t}\right\rangle}{\partial t}+r_{t} S_{t} \frac{\partial\left\langle\Pi\left(t ; S_{t}\right), X_{t}\right\rangle}{\partial s}\right. \\
& \left.+\frac{\sigma_{t}^{2} S_{t}^{2}}{2} \frac{\partial^{2}\left\langle\Pi\left(t ; S_{t}\right), X_{t}\right\rangle}{\partial^{2} s}+\sum_{j \neq C_{t-}} a_{C_{t}, j}^{\mathbb{Q}}(t)\left(\Pi_{j}\left(t ; S_{t}\right)-\Pi_{C_{t-}}\left(t ; S_{t}\right)\right)\right] d t+e^{-\int_{0}^{t}\left(r_{u}+h_{u} L_{u}\right) d u} d \mathcal{M}_{t} .
\end{aligned}
$$

As $\left\{Z\left(t, S_{t}\right)\right\}_{t \geq 0}$ is a $(\mathbb{F}, \mathbb{Q})$-local martingale, its drift must vanish. When $X_{t}=e_{i}$, this translates into the coupled system of partial differential equations given by Eq. (36).

(2) In light of the martingale property of $Z\left(t, S_{t}\right)$, we have that its dynamics must be

$$
d Z\left(t, S_{t}\right)=e^{-\int_{0}^{t}\left(r_{u}+h_{u} L_{u}\right) d u} d \mathcal{M}_{t} .
$$

Using results in Chapter 11 of Bielecki and Rutkowski (2001), we can re-write the first term of $d \mathcal{M}_{t}$ as

$$
\sum_{i=1}^{N} \sum_{j \neq i}\left[\Pi_{j}\left(t ; S_{t}\right)-\Pi_{i}\left(t ; S_{t}\right)\right] d M_{t}^{i, j}=\left\langle\Pi\left(t ; S_{t}\right), d M_{t}^{\mathbb{Q}}\right\rangle,
$$

Moreover, from the definition of $H_{t}^{i}=\mathbf{1}_{\left\{X_{t}=e_{i}\right\}}$, given in Eq. 14 , we have that the second term of $d \mathcal{M}_{t}$ can be written as

$$
\sum_{i=1}^{N} \sigma_{i} S_{t} \frac{\partial \Pi_{i}\left(t ; S_{t}\right)}{\partial s} H_{t}^{i} d W_{t}^{\mathbb{Q}}=\sigma_{t} S_{t} \frac{\partial\left\langle\Pi\left(t ; S_{t}\right), X_{t}\right\rangle}{\partial s} d W_{t}^{\mathbb{Q}}
$$

and, thus, altogether

$$
d Z\left(t, S_{t}\right)=e^{-\int_{0}^{t}\left(r_{u}+h_{u} L_{u}\right) d u}\left(\left\langle\Pi\left(t ; S_{t}\right), d M_{t}^{\mathbb{Q}}\right\rangle+\sigma_{t} S_{t} \frac{\partial\left\langle\Pi\left(t ; S_{t}\right), X_{t}\right\rangle}{\partial s} d W_{t}^{\mathbb{Q}}\right),
$$

In particular, the process $\gamma\left(t ; S_{t}\right)=e^{\int_{0}^{t}\left(r_{u}+h_{u} L_{u}\right) d u} Z\left(t, S_{t}\right)$ follows the dynamics

$$
d \gamma\left(t ; S_{t}\right)=\left(r_{t}+h_{t} L_{t}\right) e^{\int_{0}^{t}\left(r_{u}+h_{u} L_{u}\right) d u} Z\left(t, S_{t}\right) d t+\left\langle\Pi\left(t ; S_{t}\right), d M_{t}^{\mathbb{Q}}\right\rangle+\sigma_{t} S_{t} \frac{\partial\left\langle\Pi\left(t ; S_{t}\right), X_{t}\right\rangle}{\partial s} d W_{t}^{\mathbb{Q}} .
$$


Denoting $\tilde{H}(t):=1-H(t)=\mathbf{1}_{\tau>t}$, we have that the vulnerable claim price process $\Gamma\left(t, T ; S_{t}\right):=\tilde{H}(t) \gamma\left(t ; S_{t}\right)$ has dynamics

$$
d \Gamma\left(t, T ; S_{t}\right)=d\left(\tilde{H}(t) \gamma\left(t ; S_{t}\right)\right)=\tilde{H}\left(t^{-}\right) d \gamma\left(t ; S_{t}\right)+\gamma\left(t ; S_{t}\right) d \tilde{H}(t)+\Delta \gamma\left(t ; S_{t}\right) \Delta \tilde{H}(t) .
$$

where, using the hypothesis that $\Pi\left(t ; S_{t}\right)$ is continuously differentiable in $t$, and twice continuously differentiable in $S_{t}$, we have

$$
\Delta \gamma\left(t ; S_{t}\right) \Delta \tilde{H}_{t}=\left\langle\Pi\left(t ; S_{t}\right), \Delta X_{t}\right\rangle \Delta \tilde{H}_{t}=\left\langle\Pi\left(t ; S_{t}\right), \Delta X_{\tau}\right\rangle=0, \quad \text { a.s. }
$$

with the last equality being true in light of the construction (1) and the independence of $X$ and the exponential variable $\Theta$. By the Doob-Meyer decomposition of Lemma 2.2, we have $d H(t)=d \xi_{t}^{\mathbb{Q}}+\left(1-H\left(t^{-}\right)\right) h_{t} d t$ and, thus,

$$
\begin{aligned}
d \Gamma\left(t, T ; S_{t}\right)= & \left(\left(r_{t}+h_{t} L_{t}\right) \Gamma\left(t, T ; S_{t}\right)-\gamma\left(t ; S_{t}\right) h_{t} \tilde{H}\left(t^{-}\right)\right) d t+\tilde{H}\left(t^{-}\right) \sigma_{t} S_{t} \frac{\partial\left\langle\Pi\left(t ; S_{t}\right), X_{t}\right\rangle}{\partial s} d W_{t}^{\mathbb{Q}} \\
& +\tilde{H}(t)\left\langle\Pi\left(t ; S_{t}\right), d M_{t}^{\mathbb{Q}}\right\rangle-\gamma\left(t ; S_{t}\right) d \xi_{t}^{\mathbb{Q}}
\end{aligned}
$$

On the event $\tau>t$, we have $\Gamma\left(t, T ; S_{t}\right)=\gamma\left(t ; S_{t}\right)$ by definition of $\Gamma\left(t, T ; S_{t}\right)$, and also $\gamma\left(t ; S_{t}\right)=\left\langle\Pi\left(t ; S_{t}\right), X_{t^{-}}\right\rangle$. Therefore, we can write the risk-neutral dynamics of prices process $\Gamma\left(t, T ; S_{t}\right)$ as

$$
\frac{d \Gamma\left(t, T ; S_{t}\right)}{\Gamma\left(t, T ; S_{t}\right)}=\left(r_{t}+h_{t}\left(L_{t}-1\right)\right) d t+\frac{\left\langle\Pi\left(t ; S_{t}\right), d M^{\mathbb{Q}}(t)\right\rangle}{\left\langle\Pi\left(t ; S_{t}\right), X_{t-}\right\rangle}+\sigma_{t} S_{t} \frac{\frac{\partial\left\langle\Pi\left(t ; S_{t}\right), X_{t}\right\rangle}{\partial s}}{\left\langle\Pi\left(t ; S_{t}\right), X_{t^{-}}\right\rangle} d W_{t}^{\mathbb{Q}}-d \xi_{t}^{\mathbb{Q}}, \quad t<\tau,
$$

which concludes the second statement of the theorem.

(3) The price dynamics of the vulnerable claim under the real-world measure follows directly from plugging Eq. (17) into (83), and using the $\mathbb{P}$ Brownian measure defined in Eq. (35) to get:

$$
\begin{aligned}
\frac{d \Gamma\left(t, T ; S_{t}\right)}{\Gamma\left(t-, T ; S_{t-}\right)}=( & \left.r_{t}+h_{t}\left(L_{t}-1\right)+\frac{\left\langle\Pi\left(t ; S_{t}\right),\left(A^{\prime}(t)-\left(A^{\mathbb{Q}}\right)^{\prime}(t)\right) X_{t}\right\rangle}{\left\langle\Pi\left(t ; S_{t}\right), X_{t-}\right\rangle}+\frac{S_{t} \frac{\partial\left\langle\Pi\left(t ; S_{t}\right), X_{t}\right\rangle}{\partial s}}{\left\langle\Pi\left(t ; S_{t}\right), X_{t-}\right\rangle}\left(\mu_{t}-r_{t}\right)\right) d t \\
& +\frac{\left\langle\Pi\left(t ; S_{t}\right), d M^{\mathbb{P}}(t)\right\rangle}{\left\langle\Pi\left(t ; S_{t}\right), X_{t-}\right\rangle}+\sigma_{t} S_{t} \frac{\frac{\partial\left\langle\Pi\left(t ; S_{t}\right), X_{t}\right\rangle}{\partial s}}{\left\langle\Pi\left(t ; S_{t}\right), X_{t^{-}}\right\rangle} d W_{t}-d \xi_{t}^{\mathbb{P}}, \quad t<\tau,
\end{aligned}
$$

where we also used that $\xi^{\mathbb{P}}=\xi^{\mathbb{Q}}$ (see Lemma 2.2. Finally, we get the dynamics 38 since

$$
\frac{\left\langle\Pi\left(t ; S_{t}\right),\left(A^{\prime}(t)-\left(A^{\mathbb{Q}}\right)^{\prime}(t)\right) X_{t}\right\rangle}{\left\langle\Pi\left(t ; S_{t}\right), X_{t}\right\rangle}=\frac{\left\langle\left(A(t)-A^{\mathbb{Q}}(t)\right) \Pi\left(t ; S_{t}\right), X_{t}\right\rangle}{\left\langle\Pi\left(t ; S_{t}\right), X_{t}\right\rangle} .
$$

When $X_{t}=e_{i}$, the previous quantity simplifies as

$$
D_{i}\left(t, S_{t}\right):=\sum_{j=1}^{n}\left(a_{i, j}(t)-a_{i, j}^{\mathbb{Q}}(t)\right) \frac{\Pi_{j}\left(t, S_{t}\right)}{\Pi_{i}\left(t, S_{t}\right)},
$$

and 85 can be written as $\left\langle\left(D_{1}\left(t, S_{t}\right), \ldots, D_{N}\left(t, S_{t}\right)\right)^{\prime}, X_{t}\right\rangle$.

\section{Proof of Proposition 5.1.}

From the assumed time-invariance of the parameters, we have the following:

$$
\Phi_{i, m}[\Xi](\zeta)=\mathbb{E}_{i}^{\tilde{\mathbb{Q}}}\left[\Xi\left(\tilde{X}_{m}\right) e^{-\sum_{n=0}^{m} \zeta\left(U_{(n+1)}-U_{(n)}\right) \tilde{r}^{\prime} \tilde{X}_{n}-\sum_{n=1}^{m} \tilde{X}_{n-1}^{\prime} \tilde{K} \tilde{X}_{n}}\right]
$$

Let $\Lambda_{n}:=U_{(n)}-U_{(n-1)}$ for $n=1, \ldots, m+1$ and note that $\sum_{n=1}^{m+1} \Lambda_{n}=1$. It is well-known Kendall and Moran (1963) that the distribution of

$$
\Lambda:=\left(\Lambda_{1}, \ldots, \Lambda_{m}\right)
$$


is the symmetric Dirichlet distribution with parameter $\vec{\alpha}:=(1, \ldots, 1) \in \mathbb{R}^{m+1}$. We recall that a random vector $\Lambda:=\left(\Lambda_{1}, \ldots, \Lambda_{m}\right)$ follows a Dirichlet distribution with parameters $\vec{\alpha}:=\left(\alpha_{1}, \ldots, \alpha_{m+1}\right) \in \mathbb{R}^{m+1}$ such that $\min _{i} \alpha_{i}>0$ if its density is given by

$$
D\left(\lambda_{1}, \ldots, \lambda_{m}\right)=\mathbf{B}(\alpha)^{-1} \prod_{i=1}^{m} \lambda_{i}^{\alpha_{i}-1}\left(1-\lambda_{1}-\cdots-\lambda_{m}\right)^{\alpha_{m+1}-1} \mathbf{1}_{T_{m}}\left(\lambda_{1}, \ldots, \lambda_{m}\right),
$$

where $\mathbf{B}(\alpha):=\prod_{i=1}^{m+1} \Gamma\left(\alpha_{i}\right) / \Gamma\left(\sum_{i=1}^{m+1} \alpha_{i}\right)$ and $T_{m}:=\left\{\left(\lambda_{1}, \ldots, \lambda_{m}\right) \in \mathbb{R}^{m}: \lambda_{i} \geq 0 \& \sum_{i=1}^{m} \lambda_{i} \leq 1\right\}$. Next, conditioning on $\left(\widetilde{X}_{1}, \ldots, \widetilde{X}_{m}\right)$ and using independence between $U$ and $\tilde{X}$,

$$
\Phi_{i, m}[\Xi](\zeta)=\mathbb{E}_{i}^{\tilde{Q}}\left[\int_{T_{m}} e^{-\sum_{n=0}^{m} \zeta \tilde{r}^{\prime} \tilde{X}_{n} \lambda_{n+1}} D\left(\lambda_{1}, \ldots, \lambda_{m}\right) d \lambda_{1} \ldots d \lambda_{m} \Xi\left(\tilde{X}_{m}\right) e^{-\sum_{n=1}^{m} \tilde{X}_{n-1}^{\prime} \tilde{K} \tilde{X}_{n}}\right],
$$

where $\lambda_{m+1}=1-\sum_{n=1}^{m} \lambda_{n}$. The following simplification can be made:

$$
\begin{aligned}
\int_{T_{m}} e^{-\sum_{n=0}^{m} \zeta \tilde{r}^{\prime} \tilde{X}_{n} \lambda_{n+1}} D\left(\lambda_{1}, \ldots, \lambda_{m}\right) d \lambda_{1} \ldots d \lambda_{m} & =\int_{T_{m}} e^{-\sum_{n=0}^{m-1} \zeta \tilde{r}^{\prime}\left(\tilde{X}_{n}-\tilde{X}_{m}\right) \lambda_{n+1}-\zeta \tilde{r}^{\prime} \tilde{X}_{m}} D\left(\lambda_{1}, \ldots, \lambda_{m}\right) d \lambda_{1} \ldots d \lambda_{m} \\
& =e^{-\zeta \tilde{r}^{\prime} \tilde{X}_{m}} \mathcal{L}_{m}\left(\zeta \tilde{r}^{\prime}\left(\tilde{X}_{0}-\tilde{X}_{m}\right), \ldots, \zeta \tilde{r}^{\prime}\left(\tilde{X}_{m-1}-\tilde{X}_{m}\right)\right),
\end{aligned}
$$

where $\mathcal{L}_{m}$ is given as in 40 . Finally, note that, by construction, $\tilde{\mathbb{Q}}\left(\tilde{X}_{i}=\tilde{e}_{k} \mid \tilde{X}_{i-1}=\tilde{e}_{m}\right)=1 /(N-1)$ for any $k \neq m$ and, thus,

$$
\tilde{\mathbb{Q}}\left(\tilde{X}_{1}=\tilde{e}_{1}, \ldots, \tilde{X}_{m}=\tilde{e}_{m} \mid \tilde{X}_{0}=e_{i}\right)=\frac{1}{(N-1)^{m}},
$$

for all $\left(\tilde{e}_{1}, \ldots, \tilde{e}_{m}\right) \in\left\{e_{1}, \ldots, e_{N}\right\}^{m}$ such that $\tilde{e}_{i} \neq \tilde{e}_{i-1}$. In that case, it is clear that

$$
\Phi_{i, m}[\Xi](\zeta)=\sum_{\substack{\left(\tilde{e}_{1}, \ldots, \tilde{e}_{m}\right) \\ e_{i} \neq e_{i+1}}} \Xi\left(\tilde{e}_{m}\right) e^{-\zeta \tilde{r}^{\prime} \tilde{e}_{m}-\sum_{n=1}^{m} \tilde{e}_{n-1}^{\prime} \tilde{K} \tilde{e}_{n}} \mathcal{L}_{m}\left(\zeta \tilde{r}^{\prime}\left(\tilde{e}_{0}-\tilde{e}_{m}\right), \ldots, \zeta \tilde{r}^{\prime}\left(\tilde{e}_{m-1}-\tilde{e}_{m}\right)\right) \frac{1}{(N-1)^{m}}
$$

\section{Proof of Theorem 5.5 .}

Let $\mathbf{1}\left(e_{i}\right)=1$ for all $i=1, \ldots, N$, and $\|\Xi\|_{\infty}=\max _{\ell}\left|\Xi\left(e_{\ell}\right)\right|$. Let us first note that for all $u \leq v$ with $v-u \leq \delta$,

$$
\left|\widetilde{\Psi}_{i}^{(M, r)}[\Xi](u ; v)\right| \leq\|\Xi\|_{\infty} \sum_{m=0}^{M-1} e^{-(v-u)} \frac{(v-u)^{m}}{m !} \Phi_{i, m}[\mathbf{1}](v-u ; v) \leq\|\Xi\|_{\infty} \Psi_{i}[\mathbf{1}](v-u ; v) \leq\|\Xi\|_{\infty},
$$

where for the second inequality above we have use 449 ) and the fact that $\Psi_{i, m}[\mathbf{1}] \leq 1$ (being the bond price under the $i^{\text {th }}$ economic regime). In particular, from the definition (51),

$$
\begin{aligned}
\max _{i}\left|\widetilde{\Psi}_{i}^{(M, r, k)}[\Xi](j \delta ; T)\right| & =\max _{i}\left|\widetilde{\Psi}_{i}^{(M, r)}\left[\widetilde{\Xi}^{(j)}\right](j \delta ;(j+1) \delta)\right| \\
& \leq \max _{i}\left|\widetilde{\Xi}^{(j)}\left(e_{i}\right)\right|=\max _{i}\left|\widetilde{\Psi}_{i}^{(M, r, k)}[\Xi]((j+1) \delta ; T)\right| \\
& \leq \cdots \leq \max _{i}\left|\widetilde{\Psi}_{i}^{(M, r, k)}[\Xi]((k-1) \delta ; k \delta)\right| \leq\|\Xi\|_{\infty}
\end{aligned}
$$

Thus, by backward induction, it follows that

$$
\max _{j=0, \ldots, K-1} \max _{i=1, \ldots, N}\left|\widetilde{\Psi}_{i}^{(M, k, r)}[\Xi](j \delta ; T)\right| \leq\|\Xi\|_{\infty}
$$


Next, note that, for $j=k-1$ and $D:=\|\Xi\|_{\infty}(1+B)$,

$$
\begin{aligned}
\left|\widetilde{\Psi}_{i}^{(M, k, r)}[\Xi](j \delta ; T)-\Psi_{i}[\Xi](j \delta ; T)\right| & =\left|\widetilde{\Psi}_{i}^{(M, r)}[\Xi](j \delta ; T)-\Psi_{i}[\Xi](j \delta ; T)\right| \\
& \leq \sum_{m=r}^{M-1} e^{-\delta} \frac{\delta^{m}}{m !}\left|\widetilde{\Phi}_{i, m}[\Xi](\delta ; T)-\Phi_{i, m}[\Xi](\delta ; T)\right|+\sum_{m=M}^{\infty} e^{-\delta} \frac{\delta^{m}}{m !}\left|\Phi_{i, m}[\Xi](\delta ; T)\right| \\
& \leq B\|\Xi\|_{\infty} \sum_{m=r}^{M-1} e^{-\delta} \frac{\delta^{m+j}}{m !}+\|\Xi\|_{\infty} \sum_{m=M}^{\infty} e^{-\delta} \frac{\delta^{m}}{m !} \Phi_{i, m}[\mathbf{1}](\delta ; T) \\
& \leq D \delta^{(r+j) \wedge M},
\end{aligned}
$$

where we used the representation 25, 48, and the fact that

$$
\sum_{m=M}^{\infty} e^{-\delta} \frac{\delta^{m}}{m !} \Phi_{i, m}[\mathbf{1}](\delta ; T) \leq \delta^{M} \sum_{\ell=0}^{\infty} e^{-\delta} \frac{\delta^{\ell}}{\ell !} \Phi_{i, \ell}[\mathbf{1}](\delta ; T)=\delta^{M} \Psi_{i}[\mathbf{1}](T-\delta ; T) \leq \delta^{M} .
$$

Next, for $j=k-2$, using (46) and (51),

$$
\begin{aligned}
\left|\widetilde{\Psi}_{i}^{(M, k, r)}[\Xi](j \delta ; T)-\Psi_{i}[\Xi](j \delta ; T)\right| & \leq \underbrace{\left|\widetilde{\Psi}_{i}^{(M, r)}\left[\widetilde{\Xi}^{(j)}\right](j \delta ;(j+1) \delta)-\Psi_{i}\left[\widetilde{\Xi}^{(j)}\right](j \delta ;(j+1) \delta)\right|}_{T_{1}} \\
& +\underbrace{\left|\Psi_{i}\left[\widetilde{\Xi}^{(j)}\right](j \delta ;(j+1) \delta)-\Psi_{i}\left[\widehat{\Xi}^{(j)}\right](j \delta ;(j+1) \delta)\right|}_{T_{2}},
\end{aligned}
$$

where

$$
\widetilde{\Xi}^{(j)}\left(e_{i}\right):=\widetilde{\Psi}_{i}^{(M, r, k)}[\Xi]((j+1) \delta ; T), \quad \widehat{\Xi}^{(j)}\left(e_{i}\right):=\Psi_{i}[\Xi]((j+1) \delta ; T) .
$$

The term in 89 can be bounded analogously as in 88 , but replacing the constant $D$ by $\left\|\widetilde{\Xi}^{(j)}\right\|_{\infty}(1+B)$, which itself can be bounded again by $D=\|\Xi\|_{\infty}(1+B)$ in light of 87$)$. Thus,

$$
T_{1} \leq D \delta^{(r+j) \wedge M} .
$$

Similarly, we have

$$
T_{2}=\left|\Psi_{i}\left[\widetilde{\Xi}^{(j)}-\widehat{\Xi}^{(j)}\right](j \delta ;(j+1) \delta)\right| \leq \max _{\ell}\left|\widetilde{\Xi}^{(j)}\left(e_{\ell}\right)-\widehat{\Xi}^{(j)}\left(e_{\ell}\right)\right| \Psi_{i}[\mathbf{1}](j \delta ;(j+1) \delta) \leq \max _{\ell}\left|\widetilde{\Xi}^{(j)}\left(e_{\ell}\right)-\widehat{\Xi}^{(j)}\left(e_{\ell}\right)\right|,
$$

which can be bounded by $D \delta^{(r+j) \wedge M}$ in light of 88 . Putting together the previous two estimates,

$$
\left|\widetilde{\Psi}_{i}^{(M, k, r)}[\Xi]((k-2) \delta ; T)-\Psi_{i}[\Xi]((k-2) \delta ; T)\right| \leq D \delta^{(r+j) \wedge M} .
$$

Proceeding by induction, it follows that

$$
\left|\widetilde{\Psi}_{i}^{(M, k, r)}[\Xi](\ell \delta ; T)-\Psi_{i}[\Xi](\ell \delta ; T)\right| \leq(k-\ell) D \delta^{(r+j) \wedge M}=(k-\ell) D\left(\frac{T}{k}\right)^{(r+j) \wedge M},
$$

and, in particular, we obtain $(52)$ by setting $\ell=0$.

Proof of Corollary 5.6. 
The proof is very similar to that of Theorem 5.5. The key difference is in the estimate (86), which needs to be modified. Under the modified condition (53), we proceed as follows, fixing $\zeta=v-u$,

$$
\begin{aligned}
\left|\widetilde{\Psi}_{i}^{(M, r)}[\Xi](u ; v)\right| & =\left|\sum_{m=0}^{r-1} e^{-\zeta} \frac{\zeta^{m}}{m !} \Phi_{i, m}[\Xi](\zeta ; v)+\sum_{m=r}^{M-1} e^{-\zeta} \frac{\zeta^{m}}{m !} \widetilde{\Phi}_{i, m}[\Xi](\zeta ; v)\right| \\
& \leq\|\Xi\|_{\infty} \sum_{m=0}^{\infty} e^{-\zeta} \frac{\zeta^{m}}{m !}\left|\Phi_{i, m}[\mathbf{1}](\zeta ; v)\right|+\|\Xi\|_{\infty} C \sum_{m=r}^{M-1} e^{-\zeta} \frac{\zeta^{m}}{m !} \\
& \leq\|\Xi\|_{\infty} \Psi_{i}[\mathbf{1}](\zeta ; v)+\|\Xi\|_{\infty} C \zeta^{r} \\
& \leq\|\Xi\|_{\infty}\left(1+C \zeta^{r}\right) .
\end{aligned}
$$

In that case, by backward induction, Eq.877) will take the form:

$$
\max _{j=0, \ldots, K-1} \max _{i=1, \ldots, N}\left|\widetilde{\Psi}_{i}^{(M, k, r)}[\Xi](j \delta ; T)\right| \leq\|\Xi\|_{\infty}\left(1+C \delta^{r}\right)^{k}=\|\Xi\|_{\infty}\left(1+C \frac{T^{r}}{k^{r}}\right)^{k} \leq F\|\Xi\|_{\infty},
$$

for a constant $1 \leq F<\infty$ (depending only on $C, T$, and $r$ ). Following the same proof as in Theorem 5.5, we will have, by induction, that

$$
\left|\widetilde{\Psi}_{i}^{(M, k, r)}[\Xi](\ell \delta ; T)-\Psi_{i}[\Xi](\ell \delta ; T)\right| \leq(k-\ell) E \delta^{(r+j) \wedge M}=(k-\ell) E\left(\frac{T}{k}\right)^{(r+j) \wedge M},
$$

with $E:=\|\Xi\|_{\infty}(B+F)$ and Eq. (54) follows.

\section{B Pseudo-codes}

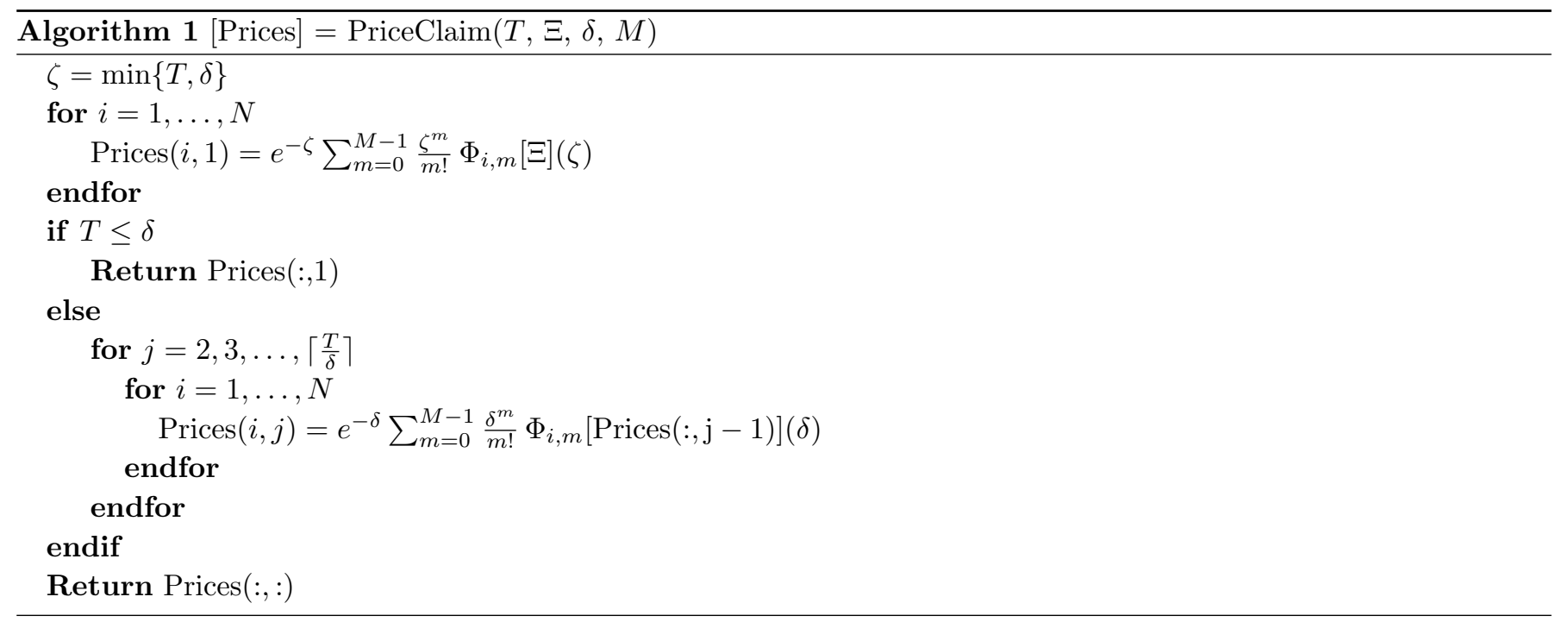

\section{Computational speed comparisons}

Table 4 compares the computational times for our proposed method (Algorithm 1) with respect to both the RungeKutta and a matrix exponential approximation method based on the representation (33) using the parameter setup of 


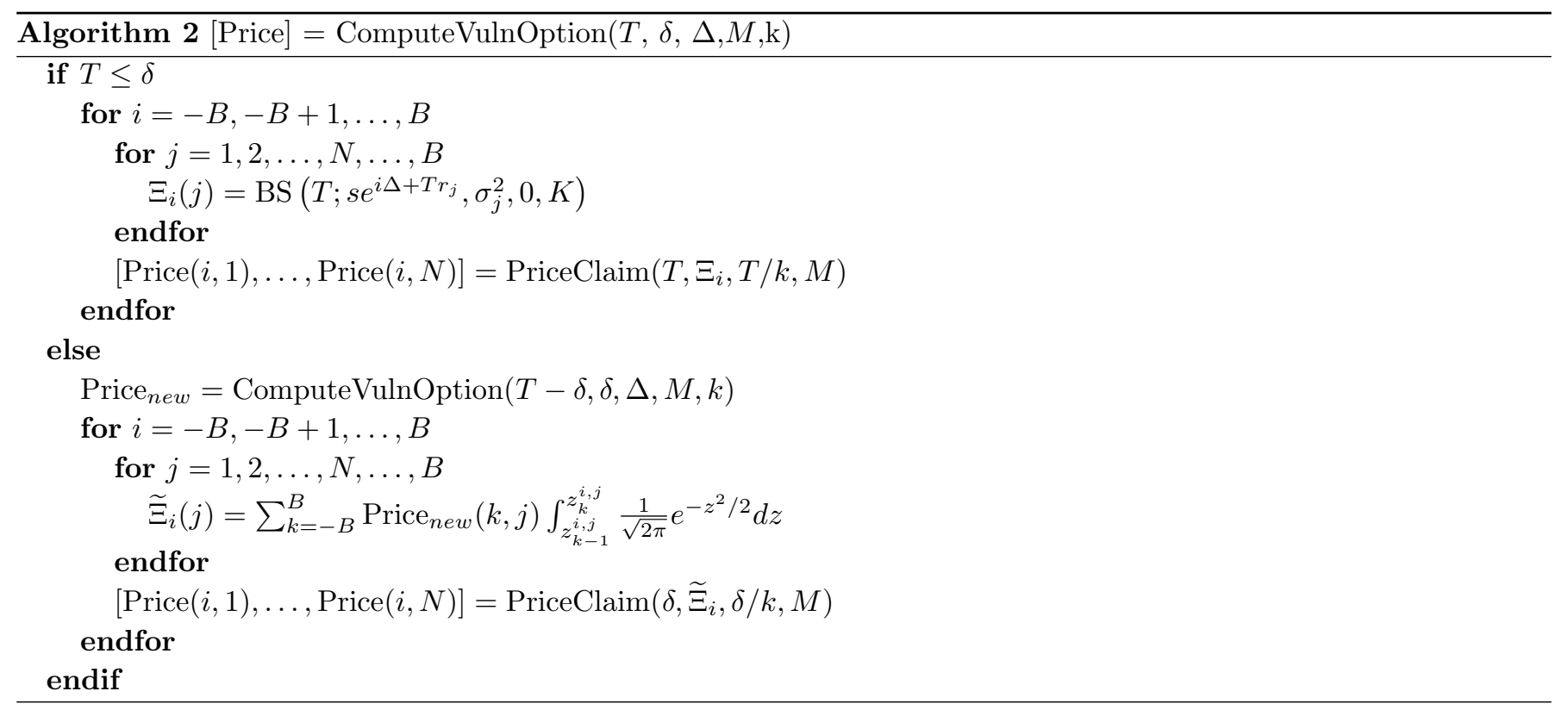

Table 1 given in Section 6.1. For each given pair $(\delta, T)$, the bond prices $\left(\psi_{1}(\zeta), \psi_{2}(\zeta), \psi_{3}(\zeta)\right)$ are computed for all timeto-maturity $\zeta \in\{\delta, 2 \delta, \ldots, k \delta:=T\}$. The entries in Table 4 represent the ratio of the new algorithm's computational time with $M=2$ to the respective ODE Method computational time and the matrix exponential computational time. As we can see, our method tends to outperform ODE Method for maturities shorter than 15 years and also for mesh sizes larger than 0.05 years; however, even in the worst case our method operates at a speed which is very comparable to the faster of the two.

\section{References}

Ang, A., and Bekaert, G. Short rate nonlinearities and regime switches. Journal of Economic Dynamics and Control 26, 7-8, 1243-1274, 2002.

Ang, A., and Bekaert, G. International Asset Allocation with Regime Shifts. The Review of Financial Studies 15, 4, $1137-1187 \mathrm{~m} 2002$.

Ang, A., and Bekaert, G. Regime switches in interest rates. Journal of Business and Economic Statistics 20, 2, 163-182, 2002 .

Belanger A., Shreve S., and Wong, D. A general framework for pricing credit risk. Mathematical Finance 14, 317-350.

Bielecki, T., and Jang, I. Portfolio optimization with a defaultable security. Asia-Pacific Financial Markets 13, 2, 113-127, 2006.

Bielecki, T., and Jeanblanc, M. Indifference prices in Indifference Pricing, Theory and

Bielecki, T.. and Crepey, S. . Dynamic Hedging of Counterparty Exposure. Forthcoming in The Musiela Festschrift, T. Zariphopoulou, M. Rutkowski and Y. Kabanov, eds, Springer.

Bielecki, T., Jeanblanc, M., and Rutkowski, M. Pricing and Hedging of Credit Risk: Replication and Mean-Variance Approaches (I). Mathematics of Finance, Proceedings of an AMS-IMS-SIAM Joint Summer Research conference on Mathematical finance, Utah conference proceedings, Yin, G. and Zhang, Q. Edtrs, 37-53, 2003. 


\begin{tabular}{|c|c|c|c|c|c|c|c|c|}
\hline \multicolumn{1}{|c|}{ Relative comp. times of new meth. vs. Runge-Kutta (N=3) } \\
\hline \multicolumn{10}{|c|}{ Time Horizons, T } \\
\hline$\delta$ & 5 & 10 & 15 & 20 & 25 & 30 & 35 & 40 \\
\hline 0.1 & 0.3093 & 0.4102 & 0.5039 & 0.5706 & 0.6701 & 0.5112 & 0.6227 & 0.7867 \\
0.075 & 0.2990 & 0.3912 & 0.4245 & 0.5749 & 0.7545 & 0.9124 & 1.1011 & 1.2094 \\
0.05 & 0.3655 & 0.4212 & 0.5828 & 0.7571 & 0.9248 & 0.8193 & 1.1855 & 1.1843 \\
0.025 & 0.4095 & 0.7270 & 1.0501 & 1.1125 & 1.5078 & 1.4490 & 1.6683 & 1.8545 \\
\hline \multicolumn{10}{|c|}{ Relative comp. times of new meth. vs. matrix exp. (N=3) } \\
\hline \multicolumn{10}{|c|}{ Time Horizons, T } \\
\hline$\delta$ & 5 & 10 & 15 & 20 & 25 & 30 & 35 & 40 \\
\hline 0.1 & 0.3828 & 0.4121 & 0.4490 & 0.5634 & 0.6183 & 0.5545 & 0.6515 & 0.7722 \\
0.075 & 0.3727 & 0.5049 & 0.6791 & 0.8080 & 0.6502 & 0.8990 & 0.8664 & 0.9054 \\
0.05 & 0.4149 & 0.7151 & 0.5571 & 0.7468 & 0.9756 & 1.0217 & 0.9247 & 1.1846 \\
0.025 & 0.5757 & 0.6770 & 0.9240 & 1.1613 & 1.0855 & 1.2903 & 1.4646 & 1.1325 \\
\hline
\end{tabular}

Table 4: The top panel shows the ratio of the processing time using our proposed method (Algorithm 1 above) and a Runge-Kutta Type numerical solution of (29) using the MATLAB function "ode45". The bottom panel shows the processing time ratio between our method and a Padé type approximation of the matrix exponential (33) using the MATLAB command "expm".

Bielecki, T., Jeanblanc, M., and Rutkowski, M. Pricing and Hedging of Credit Risk: Replication and Mean-Variance Approaches (II). Mathematics of Finance, Proceedings of an AMS-IMS-SIAM Joint Summer Research conference on Mathematical finance, Utah conference proceedings, Yin, G. and Zhang, Q. Edtrs, 54-64, 2003.

Bielecki, T., Jeanblanc, M., and Rutkowski, M. Hedging of defaultable claims Paris-Princeton Series, 126 pages, Lecture notes in Mathematical Finance, Springer. 2004.

Bielecki, T., Crepey, S., Jeanblanc, M., and Rutkowski, M. Defaultable Options in a Markov intensity model of credit risk. Mathematical Finance 18, 4, 493-518, 2008.

Bielecki, T., Crepey, S., Jeanblanc, M., and Rutkowski, M. Arbitrage Pricing of Defaultable Game Options with Applications to Convertible Bonds. Quantitative Finance 8, 8, 795-810, 2008.

Bielecki, T., and Rutkowski, M. Credit Risk: Modelling, Valuation and Hedging, Springer, New York, NY, 2001.

Blanchet-Scalliet, C., and Jeanblanc, M. Hazard rate for credit risk and hedging defaultable contingent claims. Finance and Stochastics 8, 145-159, 2004.

Bremaud, P., and Yor, M. Changes of filtration and of probability measures. Z.f.W. 45, 269-295, 1978.

Buffington, J., and Elliott, R. Regime switching and European options. In Lawrence, K.S. (ed). Stochastic theory and control. Proceedings of a Workshop, 73-81, Berline Heidelberg New York: Springer, 2002.

Capponi, A., and Figueroa-López, J.E. Dynamic Portfolio Optimization with a Defaultable Security and Regime Switching. To appear in Mathematical Finance.

Chicone, C. Ordinary Differential Equations with Applications, Springer-Verlag, New York, NY, 2006.

Coddington, E., and Levinson, N. Theory of Ordinary Differential Equations, McGraw-Hill, 1955.

Dai, Q., Singleton K.J., Yang, W. Regime shifts in a dynamic term structure model of U.S. treasury bond yields. Review of Financial Studies 20, 5, 1669-1706, 2007. 
Duffie, D., and Huang, M. Swap rates and credit quality. Journal of Finance 51, 921-949, 1996.

Duffie, J.D., and Singleton, K.J. Modeling term structures of defaultable bonds. Review of. Financial Studies 12, 687-720, 1999.

Elliott, R. J., Aggoun, L., and Moore, J. B. Hidden Markov models: estimation and control. Berlin Heidelberg NewYork: Springer, 1994.

Elliott, R.J., Chan, L., and Siu, T.K. Option pricing and Esscher transform under regime switching. Annals of Finance 1, 423-432, 2005.

Elliott, R.J., and Kopp P. Mathematics of Financial Markets. Springer, 2004.

Elliott, R. J., Wilson. C.A. The term structure of interest rates in a hidden Markov setting. In R.S. Mamon and R.J. Elliott (Ed.). Hidden Markov Models in Finance, 2007.

Elliott, R. J., and Siu, T.K. On Markov-modulated exponential affine bond price formulae. Applied Mathematical Finance, 16, 1, 2009.

Elliott, R. J., Siu, T.K., and Yang, H. Martingale representation for contingent claims with regime switching. Communications on Stochastic Analysis 1, 279-292, 2007.

Gapeev, P., and Jeanblanc, M. Pricing and filtering in a two-dimensional dividend switching model. International journal of theoretical and applied finance 13, 7, 1001-1017, 2010.

Di Graziano, G., and Rogers, L.C. Barrier Option Pricing for Assets with Markov-modulated dividends. Journal of Computational Finance 9, 4, 2006.

Hsieh, P., and Y. Sibuya. Basic Theory of Ordinary Differential Equations, Springer-Verlag, 1990.

Huge, B., and Lando, D. Swap pricing with two-sided default risk in a rating based model. European Finance Review $3,239-268$.

Kendall, M., and Moran, P. Geometrical Probability. Charles Griffin, London, 1963.

Klein, P. Pricing Black-Scholes options with correlated credit risk. Journal of Banking and Finance, 20, 1211-1129, 1996.

Giesecke, K., Longstaff, F., Schaefer, S., and Strebulaev, I. Corporate Bond Default Risk: A 150-Year Perspective. Journal of Financial Economics, 102, 2, 233-250, 2011.

Guo, X. Information and Option Pricings. Quantitative Finance 1, 28-44, 2001.

Guo, X., and Zhang, Q. Closed-form solutions for perpetual American put options with regime switching, SIAM Journal on Applied Mathematics 64, 6, 2034-2049, 2004.

Kaczorek, T. Externally and Internally Positive Time-Varying Linear Systems. Int. J. Appl. Math. Comput. Sci., 11, 4, 957-964, 2001.

Liew, C.C., and Siu, T.K. Martingale Representation and Admissible Portfolio Process with Regime Switching. Stochastic Analysis and Applications, 29, 1, 106-120, 2011.

Siu, T.K. Bond Pricing Under a Markovian Regime-Switching Jump-Augmented Vasicek Model via Stochastic Flows. Applied Mathematics and Computation, 216, 11, 3184-3190, 2010.

Siu, T.K. A Markov Regime Switching Marked Point Process for Short Rate Analysis With Credit Risk. International Journal of Stochastic Analysis, 2010. 
Siu, T.K., Erlwein, C., and Mamon, R.S. The Pricing of Credit Default Swaps Under a Markov-Modulated Merton's Structural Model. North American Actuarial Journal, 12,1, 19-46, 2008.

McNeil, A., Frey, R., and Embrechts, P. Quantitative Risk Management: Concepts, Techniques, and Tools. Princeton Series in Finance, 2006.

Norberg, R. A Markov chain Financial Market. Working Paper, University of Copenaghen/Lond School of Economics/University of Melbourne.

Yao, D., Zhang, Q., and Zhou, X.Y. A Regime-Switching Model for European Options. In: Stochastic Processes, Optimization, and Control Theory Applications in Financial Engineearing, Queueing Networks, and Manufacturing Systems, H. Yan, G. Yin and Q. Zhang (eds.), Springer, 281-300, 2006. 